\title{
A PRototype ANTI-SCATTER DETECTOR FOR MEGAVOLTAGE X-RAY IMAGing
}

\author{
by \\ Manmeet Pal Singh \\ PhD Physics, Guru Nanak Dev University, India, 2011 \\ MSc (Honors School) Physics, Guru Nanak Dev University, India, 2004 \\ BSc (Honors School) Physics, Guru Nanak Dev University, India, 2002
}

\author{
A thesis \\ presented to Ryerson University \\ in partial fulfillment of the \\ requirements for the degree of \\ Master of Science \\ in the Program of \\ Biomedical Physics \\ Toronto, Ontario, Canada, 2016 \\ (C)Manmeet Pal Singh 2016
}





\section{AUTHOR'S DECLARATION FOR ELECTRONIC SUBMISSION OF A THESIS}

I hereby declare that I am the sole author of this thesis. This is a true copy of the thesis, including any required final revisions, as accepted by my examiners.

I authorize Ryerson University to lend this thesis to other institutions or individuals for the purpose of scholarly research.

I further authorize Ryerson University to reproduce this thesis by photocopying or by other means, in total or in part, at the request of other institutions or individuals for the purpose of scholarly research.

I understand that my dissertation may be made electronically available to the public. 

A prototype anti-scatter detector for megavoltage X-ray imaging

Master of Science 2016

Manmeet Pal Singh

Biomedical Physics

Ryerson University

\begin{abstract}
In this work, a prototype anti-scatter detector based on Cherenkov radiation is developed by using glass rods. Scattering lends deleterious effects to the megavoltage x-ray portal imaging and anti-scatter detector can effectively reduce these effects. A $10 \mathrm{~cm}$ long glass rod with $1 \mathrm{~mm}$ in diameter is used as a Cherenkov detector prototype and it is studied for its response to x-ray scattering from, e.g., machine head and patient. It is subjected to $6 \mathrm{MV}$ x-ray beam generated by linear accelerator (LINAC) with different field sizes (from $3 \times 3$ to $20 \times 20 \mathrm{~cm}^{2}$ ) at different air gaps such as 10,30 and $46 \mathrm{~cm}$. The Cherenkov signal created by the detector is transmitted through optical fiber to photomultiplier tube (PMT) and measured by electrometer. The patient scattering is studied by placing a solid water phantom at isocenter. The response of single pixel Cherenkov detector is compared with the conventional ionization chamber detector.

It has been observed that glass rod based Cherenkov detector is less sensitive to scatter radiation than ion-chamber for air gap of $10 \mathrm{~cm}$. The Cherenkov signal created by glass rod is quite weak for larger air gaps and the uncertainties are quite high. Moreover, the coupling between Cherenkov detector and optical fiber is quite crucial for transmitting the Cherenkov signal from glass rod into optical fiber.
\end{abstract}





\section{Acknowledgements}

From the very first day of this project, it was clear to me that I cannot complete this research work all alone. I feel myself lucky that I have been continuously supported and helped by different people throughout this journey. I would like to take this opportunity to thank the following persons for their help and support:

First of all my esteemed supervisor, Dr. Geordi Pang, a personage of striking insight who has played a pivotal role in completion of this project. I am deeply indebted to him for his critical wisdom and passionate involvement in this research work. Working under a man of unimpeachable integrity and uncompromising principles was indeed a matter of pride for me.

My co-supervisor, Dr. Yuan Xu, an erudite scholar whose unconditional help and invaluable guidance throughout this thesis is commendable.

I am beholden to my supervisory committee member Dr. Ana Pejovic Milic, an exemplary pragmatic academician, for extending generous support and mentoring me throughout this work. I am grateful to another brilliant and dynamic intellectual Dr. Arman Sarfehnia, for his ever-encouraging support and elucidating complex concepts.

I must express my sincere gratitude to Dr. Carl Kumaradas, Program Director, Ryerson University; with his inspiration I joined the M.Sc. program in Biomedical Physics.

I am obliged to the people of Ryerson University especially Mr. Arthur Worthington, Mr. Luke Yarkaswatch, and Mr. Graham Pearson, who helped me to fabricate and design different setups required for experiments. I am also thankful to the following people from Sunnybrook hospital; Ms. Iram Munawar, Mr. Rob Etheridge, Mr. Craig McParland, and Dr. Bilal Ahmed for supporting me in carrying out experiments.

I am also thankful to Dr. Aram Teymurazyan, University of Regina, for helping me at different times during this work.

I must express my sincere thanks to my dear friends such as Mr. Ermias Woldemichael, and Mr. Aditya Pandya who supported me throughout this odyssey.

I would also like to thank Natural Sciences and Engineering Research Council of Canada (NSERC) for providing financial support for this project.

I can't express my gratitude to my beloved mother Gurjit Kaur in words, without her unconditional love and support in my life I could never have been able to complete this thesis. Her inner sanctity and unbridled joy for life has meant more to me than she will ever know.

To my wife Kamaldeep Kaur, and our children Rajmeet Singh and Sehajkamal Kaur, all I can say is it would take another thesis to express my deep love for you. Your patience, love and encouragement was a booster for me, especially in those days when I spent more time with my computer and in Lab than with you. 



\section{Contents}

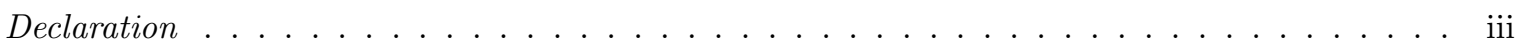

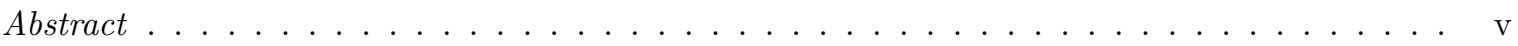

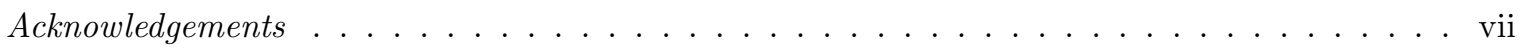

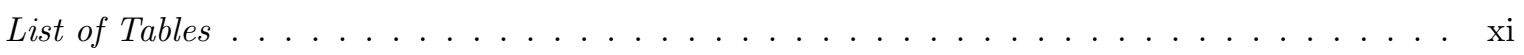

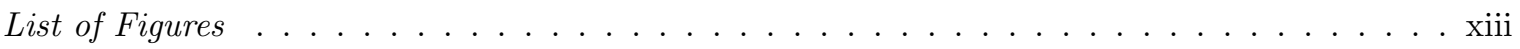

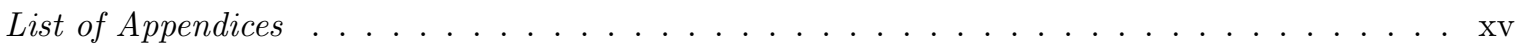

1 Introduction $\quad 1$

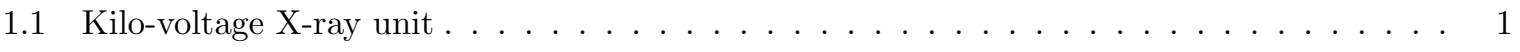

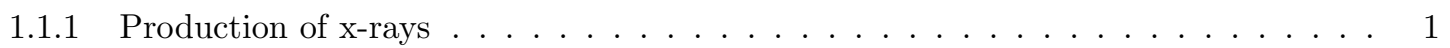

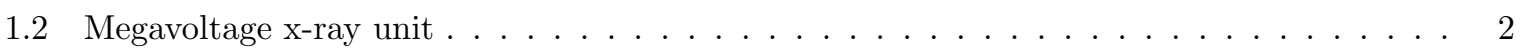

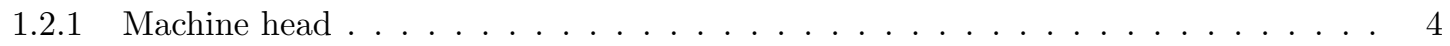

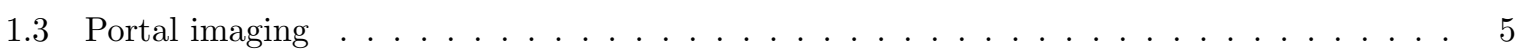

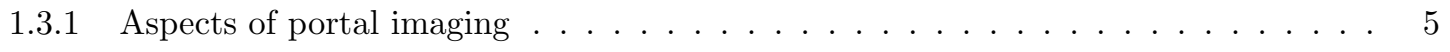

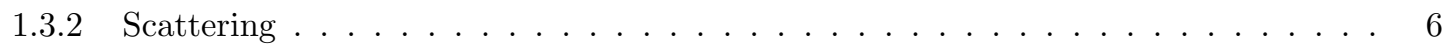

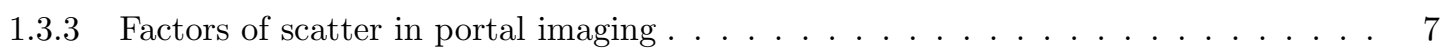

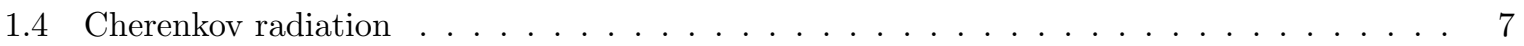

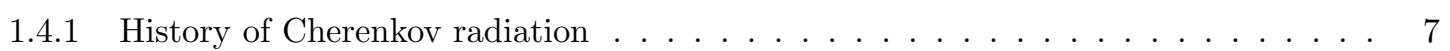

1.4 .2 Theory of Cherenkov effect $\ldots \ldots \ldots \ldots \ldots \ldots$

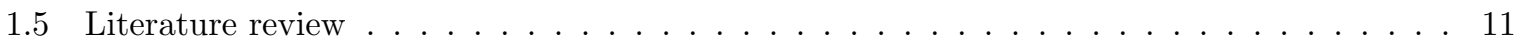

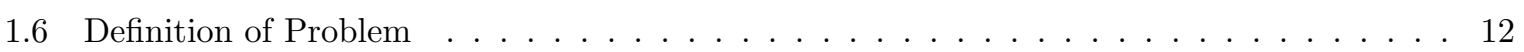

1.6 .1 Hypothesis . . . . . . . . . . . . . . . . . . . 13

1.6 .2 Specific objectives . . . . . . . . . . . . . . . . 13

2 Experimental Methodology 15

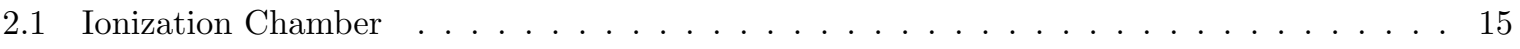

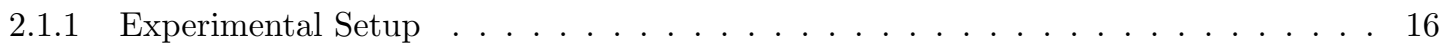

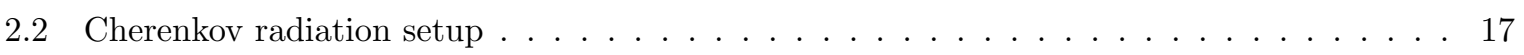

2.2 .1 Single Glass $\operatorname{rod} \ldots \ldots \ldots \ldots \ldots \ldots \ldots$

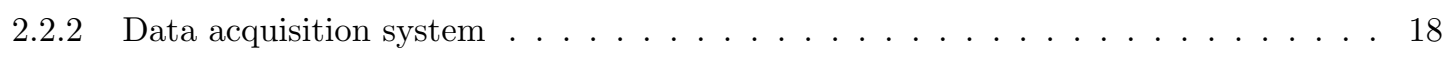

2.2 .3 Cherenkov radiation from group of glass rods $\ldots \ldots \ldots \ldots$ 
3 Results and Discussion $\quad 21$

3.1 Results of Ionization Chamber f . . . . . . . . . . . . . . . . . . . 21

3.1.1 Ionization Chamber placed in air . . . . . . . . . . . . . . . . . . . 21

3.1 .2 Ionization Chamber in Glass slab . . . . . . . . . . . . . . . . . . . . . . . 24

3.2 Results of Cherenkov Radiation . . . . . . . . . . . . . . . . . . . . 30

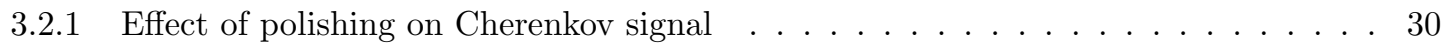

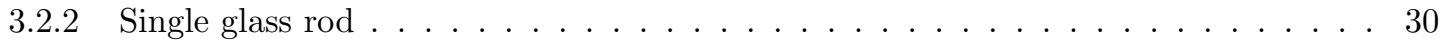

3.2 .3 Group of glass rods . . . . . . . . . . . . . . . . . . . . . . . 33

4 Conclusion $\quad 39$

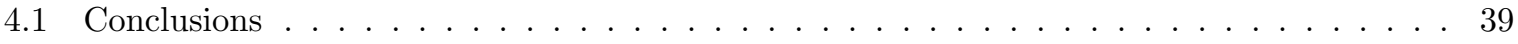

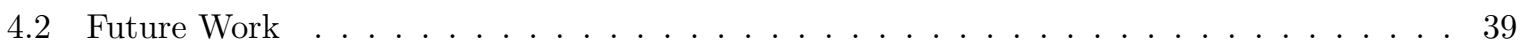

$\begin{array}{ll}\text { Appendices } & 41\end{array}$

$\begin{array}{ll}\text { References } & 45\end{array}$

$\begin{array}{ll}\text { Index } & 47\end{array}$ 


\section{List of Tables}

1.1 The values of parameters of Cherenkov radiation in water and dry air . . . . . . . . 11

3.1 Primary signal obtained after extrapolated the curves represented in figures 3.1 to 3.3 and 3.7 to 3.927

3.2 Comparison of average total signal measured by ionization chamber when placed in air and glass slab 28

3.3 Comparison of scatter fraction measured by ionization chamber when placed in air and glass slab 28

3.4 Primary signal from Cherenkov radiation obtained after extrapolated the curves . . . . . 31 



\section{List of Figures}

1.1 Diagram of diagnostic x-ray tube(Adapted from Khan's Physics of Radiation Therapy) . . 2

1.2 Spatial distribution of emitted x-rays (Adapted from Khan's Physics of Radiation Therapy) 3

1.3 Block diagram of clinical LINAC (Adapted from Khan's Physics of Radiation Therapy) 3

1.4 Different components of LINAC head (Adapted from Khan's Physics of Radiation Therapy) 4

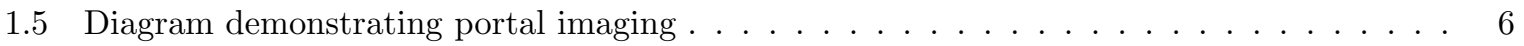

1.6 Polarization with the passage of electron at (a) low velocity (b) high velocity (Adapted from J.V. Jelly) 8

1.7 Huygens construction to explain coherence (Adapted from J.V. Jelly) _ . . . . . . . . . 9

1.8 Formation of Cherenkov radiation cone (Adapted from J.V. Jelly) . . . . . . . . . . . 10

1.9 Schematic of CPID (Adapted from Teymurazyan and Pang (2012)) . . . . . . . . . . . 12

2.1 Schematic of measurement with ionization chamber in glass slab . . . . . . . . . . 17

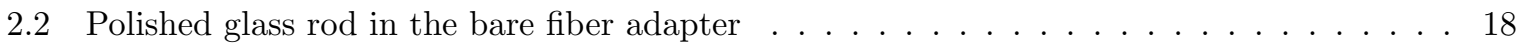

2.3 Effect of polishing on the surface of end of glass $\operatorname{rod} \ldots \ldots \ldots \ldots$

2.4 Schematic of experimental setup with data acquisition $\ldots \ldots \ldots \ldots \ldots \ldots$

3.1 Electrometer readings by ionization chamber placed in air, at air gap $10 \mathrm{~cm} \ldots \ldots$. . . 22

3.2 Electrometer readings by ionization chamber placed in air, at air gap $30 \mathrm{~cm}$. . . . . . . 22

3.3 Electrometer readings by ionization chamber placed in air, at air gap $46 \mathrm{~cm} \ldots \ldots$. . . 23

3.4 Scatter fraction by ion-chamber placed in air, at air gap $10 \mathrm{~cm} \ldots \ldots \ldots$. . . . . . 24

3.5 Scatter fraction by ion-chamber placed in air, at air gap $30 \mathrm{~cm} \ldots \ldots \ldots \ldots$

3.6 Scatter fraction by ion-chamber placed in air, at air gap $46 \mathrm{~cm} \ldots \ldots \ldots \ldots$

3.7 Electrometer readings by ion-chamber placed in glass slab, at air gap $10 \mathrm{~cm} \ldots \ldots$. . . . 26

3.8 Electrometer readings by ion-chamber placed in glass slab, at air gap $30 \mathrm{~cm} \ldots \ldots$. . . . 26

3.9 Electrometer readings by ion-chamber placed in glass slab, at air gap $46 \mathrm{~cm} \ldots \ldots$. . . . 27

3.10 Scatter fraction by ion-chamber placed in glass slab, at air gap $10 \mathrm{~cm} \ldots \ldots$. . . . . . 29

3.11 Scatter fraction by ion-chamber placed in glass slab, at air gap $30 \mathrm{~cm} \ldots \ldots$. . . . . . 29

3.12 Scatter fraction by ion-chamber placed in glass slab, at air gap $46 \mathrm{~cm} \ldots \ldots$

3.13 Cherenkov signal by unpolished rod placed in glass slab, at air gap $10 \mathrm{~cm}$. . . . . . . 31

3.14 Cherenkov signal by polished glass rod placed in glass slab, at air gap $10 \mathrm{~cm}$. . . . . 32

3.15 Cherenkov signal by polished rod placed in glass slab, at air gap $30 \mathrm{~cm}$. . . . . . . . . 32

3.16 Cherenkov signal by polished rod placed in glass slab, at air gap $46 \mathrm{~cm} \ldots \ldots$. . . . . . 33 
3.17 Scatter fraction by polished rod, at air gap $10 \mathrm{~cm} \ldots \ldots \ldots \ldots \ldots$

3.18 Scatter fraction by polished rod, at air gap $30 \mathrm{~cm} \ldots \ldots \ldots \ldots \ldots$

3.19 Scatter fraction by polished rod, at air gap $46 \mathrm{~cm} \ldots \ldots \ldots \ldots$

3.20 Cherenkov radiation from group of rods, at air gap $10 \mathrm{~cm} \ldots \ldots \ldots \ldots$

3.21 Cherenkov radiation from group of rods, at air gap $30 \mathrm{~cm} \ldots \ldots \ldots$

3.22 Cherenkov radiation from group of rods, at air gap $46 \mathrm{~cm} \ldots \ldots \ldots$ 


\section{List of Appendices}





\section{Chapter 1}

\section{Introduction}

X-rays were discovered by Wilhelm Conrad Roentgen in 1895, when he was working with cathode rays in a discharge tube. He named these new rays as x-rays, he observed that these rays were capable of penetrating opaque materials, creating fluorescence, blackening photographic plate, and ionizing a gas. It was one of the revolutionizing discoveries of $19^{\text {th }}$ century, within a few months of discovery medical doctors adopted x-ray for diagnosis (3). These x-rays proved to be most utilitarian radiation which are applied in different fields such as medical radiography, radiation therapy, microscopy, crystallography, etc. These different applications utilize x-rays of different energies and the present work utilizes x-rays in radiation therapy regime. In this chapter, all the concepts used in present thesis are introduced briefly. In the following section, production and scattering of x-rays are discussed briefly.

\subsection{Kilo-voltage X-ray unit}

The x-rays are produced whenever a metal substance is bombarded by high speed electrons, and diagnostic Kilo-voltage x-rays are produced in the evacuated glass tube. The x-ray tube consists of a cathode at one end and anode at other end, cathode assembly has a filament of tungsten placed inside a focusing cup and anode has a target made of tungsten or a tungsten rhenium alloy. On heating the filament, the electrons are emitted from filament due to thermionic emission, these electrons are attracted towards anode because it is kept at high positive with respect to filament. These fast moving electrons strike the target at anode and due to deceleration or stopping of electrons the x-rays are produced and beam emerges through glass window or beryllium window (15) and (16). The x-ray tube is represented in figure 1.1.

\subsubsection{Production of x-rays}

The x-ray production can be explained on the basis of two mechanisms namely Bremsstrahlung x-rays and Characteristic x-rays. Bremsstrahlung means 'braking radiation' is the process of producing $\mathrm{x}$-rays 


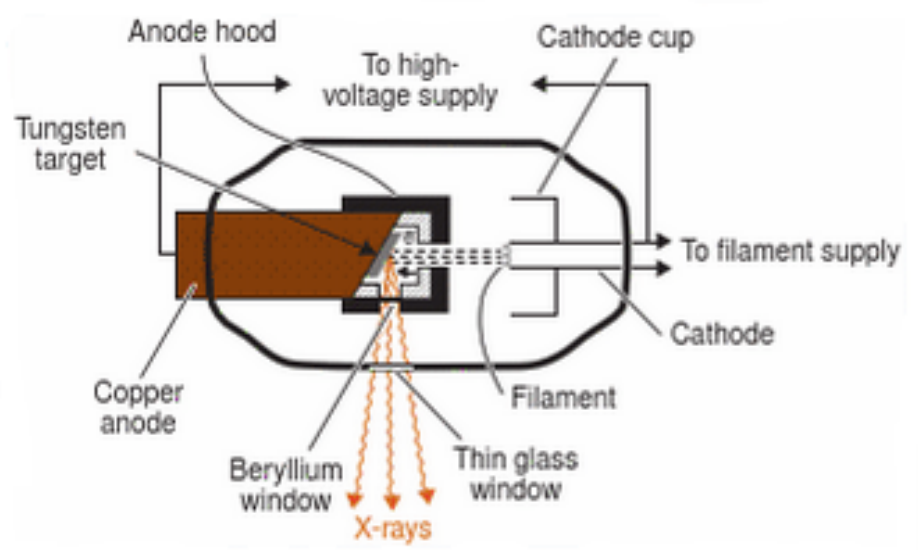

Figure 1.1: Diagram of diagnostic x-ray tube(Adapted from Khan's Physics of Radiation Therapy)

as a result of radiative collision between high speed electron and a nucleus. An electron can have one or more bremsstrahlung interactions in a material, and an interaction may result in partial or complete loss of energy of electron. The resulting bremsstrahlung photon may have any energy up to the energy of incident electron, moreover the direction of emitted photon also depends on energy of incident electron, spatial distribution of x-rays is represented in figure 1.2 (16), (15). At electron energies below $100 \mathrm{keV}$, $\mathrm{x}$-rays are emitted in all directions equally, with the increase in incident energy of electrons the emission of x-ray photon becomes increasingly forward directed. Bremsstrahlung x-rays have spectrum of energies where maximum energy is equal to peak voltage and average energy is approximately equal to one-third of incident electron (16), (15).

On other hand, characteristic x-rays are produced when incident electrons knock out electron from the $\mathrm{K}, \mathrm{L}$ or $\mathrm{M}$ orbital of target material and vacancy is being created. The electron from outer orbital falls in to fill the vacancy, as a result energy is emitted in the form of electromagnetic radiation called characteristic x-ray. It is called characteristic because it is characteristic of the transition between orbitals of the target atom (16), (15).

\subsection{Megavoltage x-ray unit}

The x-ray beams of energy 1 MV or greater are termed as megavoltage beams, for generating such a high energy beam clinically accelerators are employed. The linear accelerator (LINAC) is a device that uses high-frequency electromagnetic waves to accelerate charged particles such as electrons to high energies through an accelerator tube. There are two types of accelerator structures employed for radiation therapy; stationary or traveling wave guides structures. The block diagram of structure of linear accelerator is presented in figure 1.3. Power supply provides direct current (DC) power to modulator and it creates high voltage pulses which are delivered to magnetron or klystron and electron gun simultaneously. The 


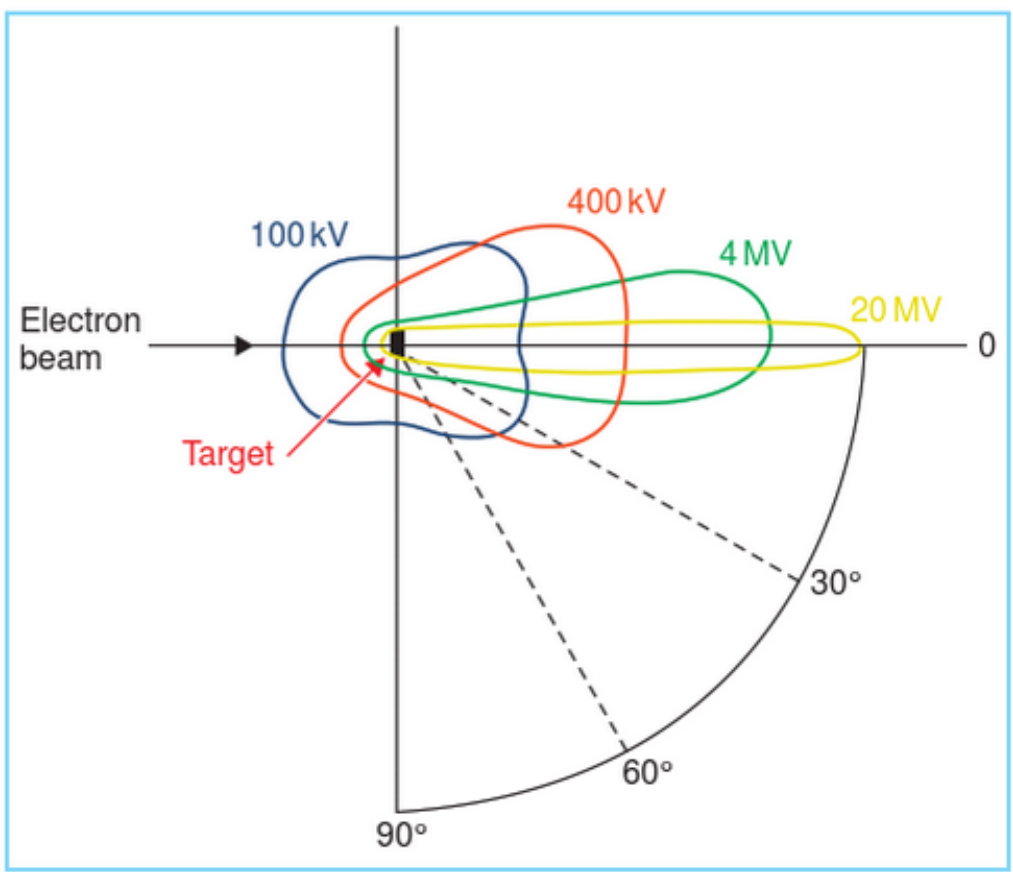

Figure 1.2: Spatial distribution of emitted x-rays (Adapted from Khan's Physics of Radiation Therapy)

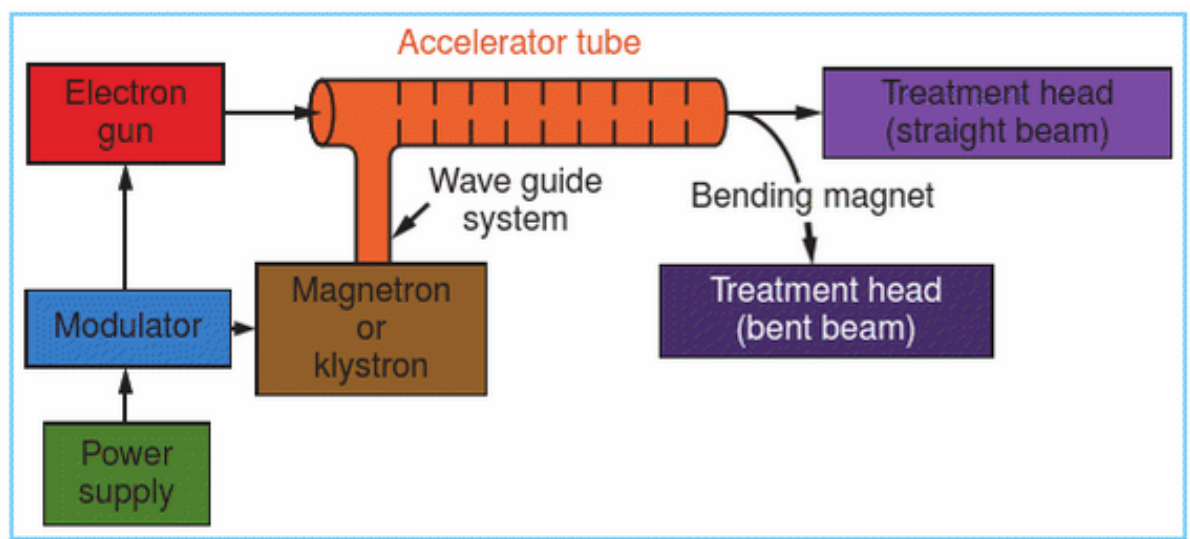

Figure 1.3: Block diagram of clinical LINAC (Adapted from Khan's Physics of Radiation Therapy) 
magnetron or klystron creates a pulsed microwave which are injected in to accelerator tube through wave guide system and at proper instant electron gun injects electrons into evacuated accelerator structure. The electrons enter accelerator tube with initial energy of $50 \mathrm{keV}$, they get accelerated to high energy by electromagnetic field of microwaves. The high energy electron exit accelerator structure in the form of pencil beam of $3 \mathrm{~mm}$ diameter. Subsequently, electrons are bent through the angles from 90 to 270 degrees through bending magnets, focusing coils and other components. Resulting beam is allowed to strike the target to produce Bremsstrahlung x-rays (16), (15).

Bremsstrahlung $\mathrm{x}$-rays are produced when electrons strike the high-z target material, it is water cooled and thick enough to absorb the incident electrons. As a result of Bremsstrahlung, the produced $\mathrm{x}$-rays have spectrum of energies with maximum energy equal to incident energy. If the energy of striking electron beam is $6 \mathrm{MeV}$, the resulting photon spectrum is named as $6 \mathrm{MV}$. The average photon energy of the beam is approximately one-third of the maximum energy (16), (15).

The electron beam exiting the accelerator tube as a pencil beam is also used for treatment in electron mode of LINAC. In electron mode of operation the electron beam passes through scattering foil instead of target, this spreads the electron beam and to get a uniform fluence across the treatment field (16).

\subsubsection{Machine head}

The machine head is a vital part of medical LINAC, it constitutes number of components and accessories, main parts of head are x-ray target, scattering foil, flattening filter, ion chamber, fixed and moving collimator, and light localizer system. The arrangement of different components in machine head are shown in figure 1.4. The electron beam is collimated by fixed primary collimator then high energy electron
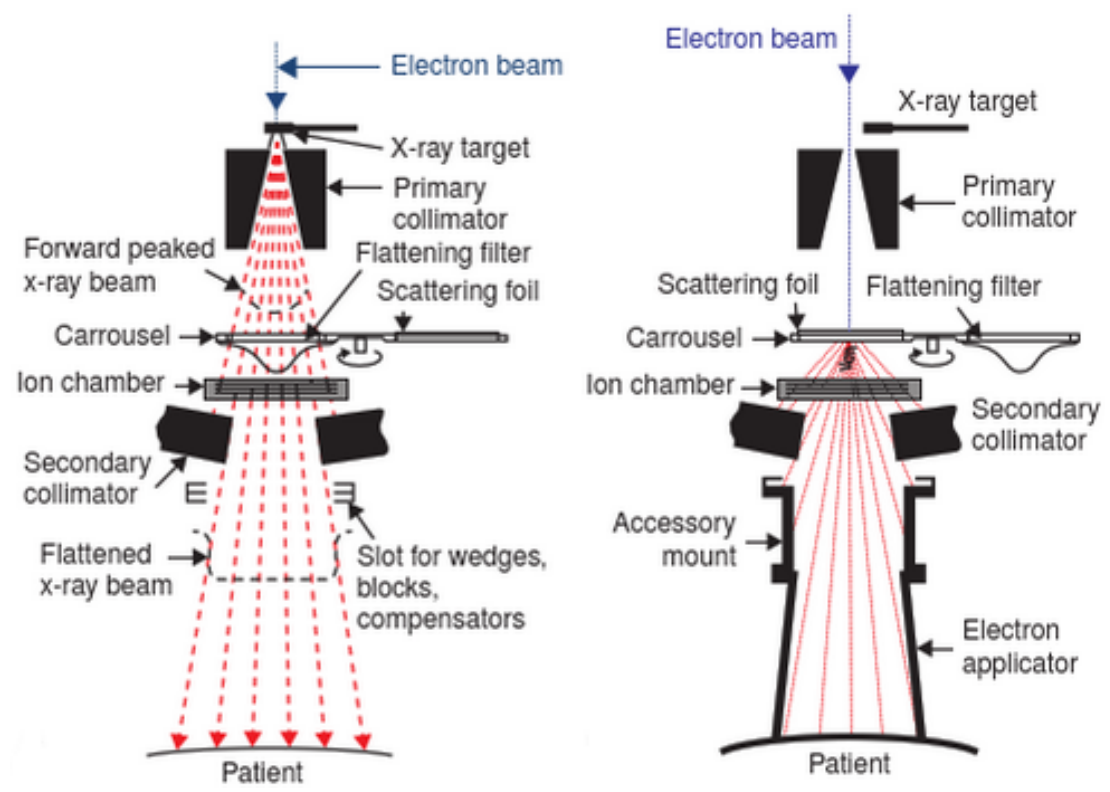

Figure 1.4: Different components of LINAC head (Adapted from Khan's Physics of Radiation Therapy) 
beam passes through the target and generates x-ray, the peak of intensity is in forward direction. In order to make intensity uniform across the beam, the flattening filter made up of lead or tungsten is inserted in the beam. The beam emerging out of flattening filter is incident on dose monitoring chambers which monitor dose rate, integrated dose, and field symmetry (16).

After passing through ion chambers, beam is further collimated by moving collimator made up of tungsten or lead blocks. These collimators can project the beam in various shapes with size varying from nearly $0 \times 0$ to $40 \times 40 \mathrm{~cm}^{2}$ at $100 \mathrm{~cm}$ from the x-ray source. The field size is confirmed by the system of light source and mirrors located in treatment head, and this light field is congruent with radiation field (16).

The linear accelerators have capabilities in which gantry and x-ray source can rotate about a horizontal axis, whereas collimator axis, which is coincident with central axis of beam, can move in vertical axis. The point of intersection of collimator axis and axis of rotation of gantry is called (mechanical) isocentre (16).

\subsection{Portal imaging}

Using the therapeutic x-ray beam, images of treatment area being irradiated can be made, this process is called portal imaging. The major application of portal images has been the study of set-up errors in patient treatment and quantification of uncertainties in accounting for margins considered while treatment. The therapeutic beam have energies in the megavoltage range, therefore Compton scattering is the predominant mechanism of photon interaction. The Compton interaction is almost independent of atomic number $Z$, therefore portal imaging inherent less contrast as compared to diagnostic x-ray (17).

The efforts has been continuously underway to enhance the quality of portal imaging and ease of acquisition (17), (21), (10), (5). As a result of research and development, portal imaging devices are becoming able to give both geometrical and dosimetric information (17).

\subsubsection{Aspects of portal imaging}

According to Boyer et al (5) radiation beam that exits patient has a lot of information which can be extracted. The quality of detector can be determined in terms of detector quantum efficiency (DQE), and can be defined as efficiency of detector to covert incident x-ray into image signal (17). DQE depends on various parameters such as efficiency of detection of incident beam, spatial resolution of the system and system noise. By increasing the detection efficiency of system and reducing the noise image quality can be enhanced (5). The information contained in image depends on signal to noise ratio (SNR), in turn SNR depends on the size of structure of interest, contrast and number of x-ray quanta used to form image (17). The contrast of megavoltage portal image is 10-20 times lower than that of diagnostic x-ray imaging, therefore for the same SNR between diagnostic and megavoltage imaging, large number of photons are required for portal imaging (17). Hence for diagnostic quality portal imaging high DQE is required, other factors which effect the portal imaging are magnification and scattered radiation (17). For portal imaging, optical magnification is between about 1 and 2, depending on medium of imaging 


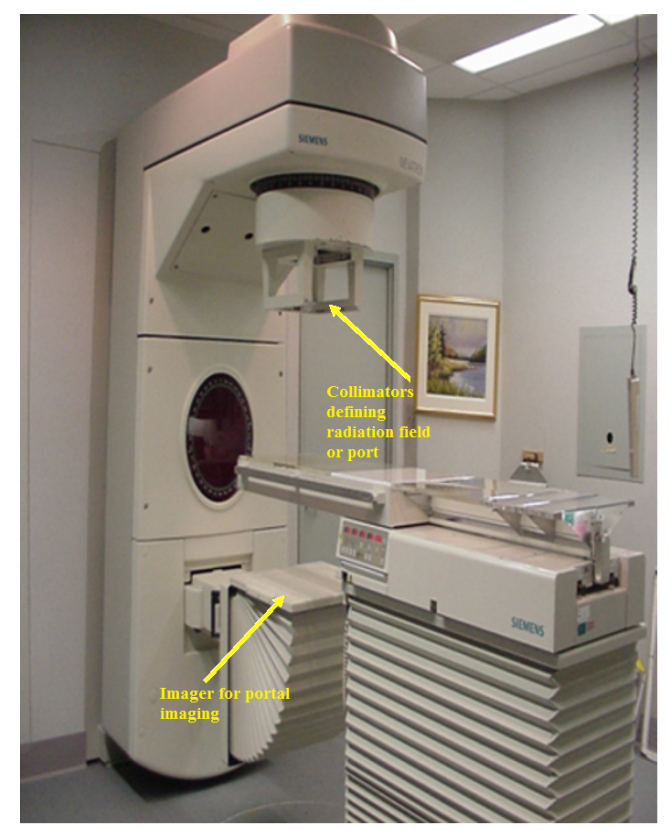

Figure 1.5: Diagram demonstrating portal imaging

(4), (2). The image quality is degraded by scattered radiation and for diagnostic imaging anti-scatter grid has been effectively used but for megavoltage imaging it is not feasible because the energy of scattered beam is quite high (18), (2), (12). The anti-scatter grid for MV gamma rays has been prepared by Watson et al (27), and grid weighs almost 1 ton which makes it impractical for usage in medical LINAC.

\subsubsection{Scattering}

When x-rays interact with tissue made up of different materials such as muscle, bone, etc. it get attenuated differentially and it provides contrast to the image. The part of X-rays passing straight without interactions with tissue are referred to as primary radiation. Those $\mathrm{x}$-rays that alter their trajectory between source and detector due to interaction with medium are called secondary radiation or scattered radiation. If X-rays get absorbed completely and not reach the detector they are called absorbed radiation. In the diagnostic radiology that is in energy range of 25-150 keV, X-rays interact with tissue predominantly through coherent, photoelectric and Compton effects. Photoelectric interactions result in X-ray absorption, whereas coherent and Compton effects result in scatter radiation (28).

In radiotherapy beam energies, $\mathrm{x}$-rays interact mainly through Compton effect which is independent of atomic number of material and provides poorer image contrast to portal imaging (28). 


\subsubsection{Factors of scatter in portal imaging}

Since scattering leads deleterious effect to portal imaging, the main factors that effect scattering of x-ray beam in portal imaging or in radiation therapy are (12):

1. Phantom (patient) thickness: According to Jaffray et al (12) with the increase in phantom thickness, the transmission of primary beam to the detector decreases, whereas scatter fluence is not much effected. It was reported that constant increase in scatter fraction with increase in thickness was mainly due to the decrease in transmission of primary beam (12).

2. Air gap: Air gap is the distance between phantom and detector, with the increase in air gap scatter fluence reduces. Scatter beam reduces more rapidly than primary beam which follows inverse square, because scattered beam has broader angular distribution (12). It not only reduces the scatter photons reaching the detector but also hardens the scatter spectra by filtering low energy scattered photons (12).

3. Field size: Field size is the rectangular opening provided by collimator ranging from $0 \times 0$ to $40 \times 40 \mathrm{~cm}^{2}$ projected at distance of $100 \mathrm{~cm}$ from the x-ray source (16). The fluence of primary beam is independent and not effected by the field size, it remains same for all the field sizes, only the scatter fraction increases with the increase in field size (12).

\subsection{Cherenkov radiation}

The scattering of MV x-ray beam reduces the quality of portal imaging and this problem of scattering can be addressed on the basis of Cherenkov radiation. Before discussing the present problem and its plausible solution; it is imperative to explain basics of Cherenkov radiation briefly.

\subsubsection{History of Cherenkov radiation}

In the earlier days of radioactivity, bluish-white light was observed in the solution of strong sources of beta and gamma particles, at that time there was no reasonable explanation for this effect(13). Only two properties of this light were known at that time: firstly colour and intensity of light were independent of medium, secondly, the spectrum of this visible light is continuous. First serious attempt to explain continuous spectra was made by Mallet. He published three papers but unfortunately his work was not carried out further experimentally or theoretically (13).

A few years later Cherenkov carried out a long series of experiments independently to explain this phenomena (6). He proved conclusively that this light is created due to passage of fast moving electrons through a transparent medium. He also discovered unique polarization and directional properties of these radiation. The considerable amount of work was carried out by Cherenkov on these radiation, hence these were named after him (13). At the same time Frank and Tamm explained this effect theoretically on the basis of classical electrodynamics (13). After 1945, research in this field got an impetus, especially with the discovery of photomultiplier tubes which can detect the faint pulses of light. Moreover, soon 
Cherenkov radiation were also employed as a radiation detector, the unique properties of this radiation enable it to distinguish between different types of particles and measure their velocities directly (13).

\subsubsection{Theory of Cherenkov effect}

Consider an electron traveling relatively slow through the glass tube or any transparent material as shown in figure 1.6. In the normal state, atoms of the glass are roughly spherical in shape, but with the passage of electron the shape of atom will get distorted due to electric field created by it. The atoms will behave as dipoles and the negative charge in atom will get displaced away from the track of electron and to one end of positive charge. Therefore, the material gets polarized in the space around the traveling electron and each elemental space in material will release a short electromagnetic pulse. After electron has passed by, the polarized atoms of the material reverts back to their normal shape. The polarization around the track of electron is highly symmetrical therefore no field or radiation at large distance from the path of electron (13), (14).

If the electron is traveling with very high velocity comparable to the speed of light in the medium,
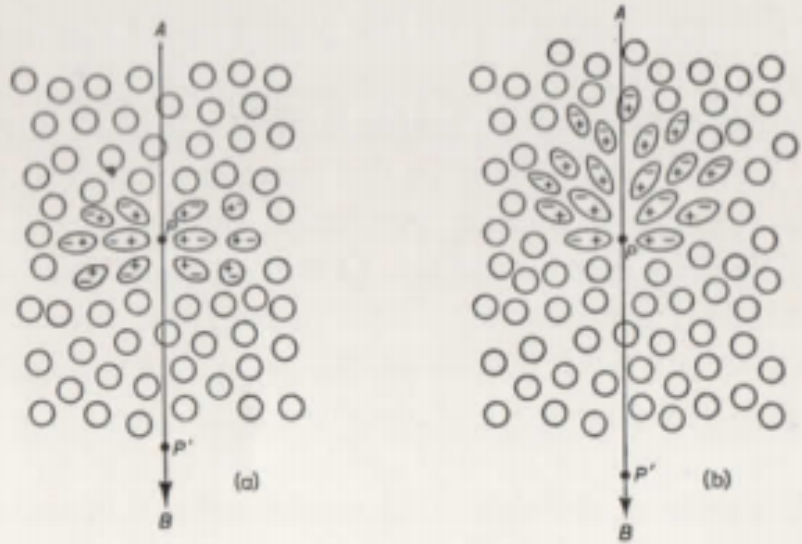

Figure 1.6: Polarization with the passage of electron at (a) low velocity (b) high velocity (Adapted from J.V. Jelly)

the polarization is not symmetrical. In azimuthal plane polarization is symmetrical whereas along the axis resultant polarization is observed even at longer distance from the track. Due to the electric field these elements will radiate electromagnetic pulses with overlapping frequencies. The phase velocity of electron is greater than that of light and the radiated pulses along the track are in phase. These coherent pulses are emitted in the form of wavelets on wavefront inclined at an angle $\theta$ along the path of electron (13), (14). These coherent wavelets are explained on the basis of Huygen's construction as shown in figure 1.7. Along the path length $\mathrm{AB}$, pulses are emitted from different points $P_{1}, P_{2}$ and $P_{3}$ at angle $\theta$ and these wavelets form a plane wavefront BC. Since the particle travels distance AB and emitted light travels distance AC in the same time, therefore emitted pulses are coherent. If $c$ is the speed of light in vacuum, then its velocity in a medium, with a refractive index of $n$, becomes $\frac{c}{n}$. The velocity of particle 


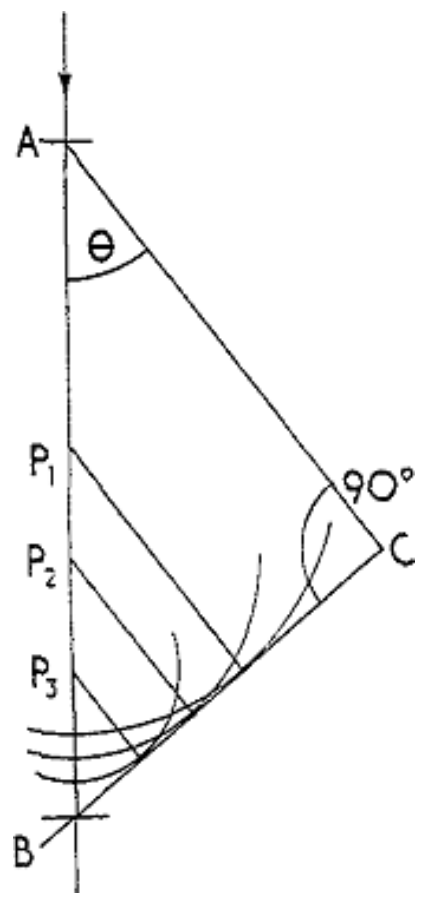

Figure 1.7: Huygens construction to explain coherence (Adapted from J.V. Jelly)

in the medium can be calculated from relativistic relation $\beta c$ (14). Let $\delta t$ be time taken by particle to travel distance $\mathrm{AB}$, therefore,

$$
A B=\beta c . \delta t
$$

where $\beta=\frac{v}{c}$ is relativistic velocity calculated from kinetic energy and rest mass of the particle which is given by following equation:

$$
E=m c^{2}\left[\frac{1}{\sqrt{1-\beta^{2}}}-1\right]
$$

Now the distance AC traveled by light is calculated as

$$
A C=\left(\frac{c}{n}\right) \delta t
$$

Since $\mathrm{ABC}$ is right angled triangle, and using the above two relationships

$$
\cos \theta=\frac{A C}{A B}=\frac{\left(\frac{c}{n}\right) \delta t}{\beta c . \delta t}
$$

therefore,

$$
\cos \theta=\frac{1}{\beta n}
$$


This relationship is called "Cherenkov relation" (13), (14).

The observations that come out of this relationship are (6) (13), (14):

1. Threshold, critical, or minimum velocity of a particle in a medium below which no radiation is created, is the case when $\theta$ becomes zero. This means that at critical velocity, emitted radiation and particle travel in same direction. Mathematically, $\beta_{\min }=\frac{1}{n}$.

2. For a particle traveling with ultra-relativistic velocity, $\beta=1$, particle and emitted radiation travel with maximum angle that is $\theta_{\max }=\cos ^{-1}(1 / n)$.

Thus, Cherenkov effect is analogous of the "supersonic bang" (13). The light is emitted on a conical surface whose axis coincides with the path of traveling particle, and the semi-apex angle is $\theta$. It is represented in figure 1.7. Cherenkov radiation has unique polarization properties in which electric

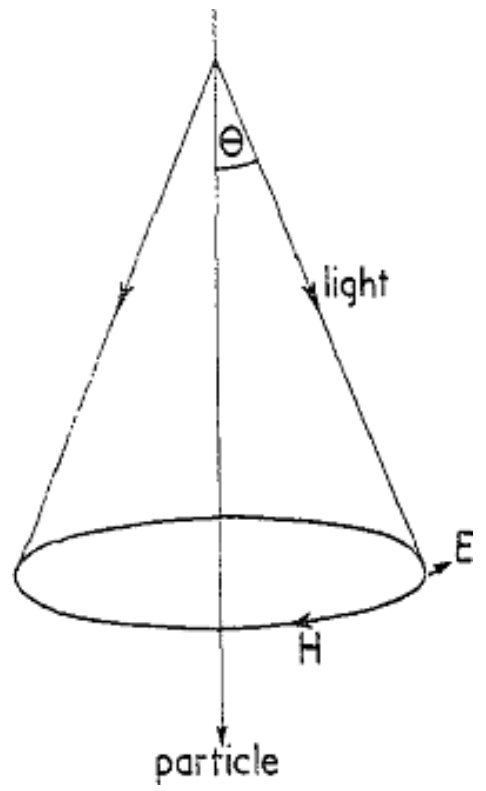

Figure 1.8: Formation of Cherenkov radiation cone (Adapted from J.V. Jelly)

vector $\mathrm{E}$ is perpendicular to the surface of cone and magnetic vector $\mathrm{H}$ is along the tangent to this cone (14), (13).

Frank and Tamm (13), worked on calculating the amount of light (photons) produced per unit length by a particle with charge e, creating light with angular frequency $\omega$, this is calculated by the relationship:

$$
\frac{d W}{d l}=\frac{e^{2}}{c^{2}} \int_{\beta n>1} \omega d \omega\left(1-\frac{1}{\beta^{2} n^{2}}\right)
$$

In the Table 1.1, numerical values of different parameters in air and water, calculated by J.V. Jelly (13) are presented. The values of different parameters of Cherenkov radiation strongly depend on the medium in which charged particle is traversing. These radiation have a lot of applications which are discussed 


\begin{tabular}{|c|c|c|}
\hline Parameter & Water & Air (N.T.P) \\
\hline \hline Refractive index $(\mathrm{n})$ & 1.33 & 1.00029 \\
$\theta_{\max }($ when $\beta \rightarrow 1)$ & $41^{\circ}$ & $1.3^{\circ}$ \\
Threshold energies $E_{T}$ when $\theta=0$ & $260 \mathrm{keV}$ electrons & $21 \mathrm{MeV}$ \\
$\frac{d W}{d l}$ (photons/cm, between $\left.3500-5500 \AA\right)$ & 250 & 0.3 \\
\hline \hline
\end{tabular}

Table 1.1: The values of parameters of Cherenkov radiation in water and dry air

by Jelly (14), (13). In this work only application of Cherenkov radiation in portal imaging is discussed. Development and application of Cherenkov radiation based imaging devices are explained in the section of literature review.

\subsection{Literature review}

Cherenkov radiation has numerous applications in the detection and counting of nuclear and cosmic rays (14), (13). In clinical application, Cherenkov radiation has been considered as a noise in scintillation dosimeters (20), (7), the work of using Cherenkov for imaging and dosimetry has been carried out extensively by research groups of Geordi Pang et al and Brain Pogue et al. The work carried out by these groups are discussed in the following paragraphs.

One of the most important requirement in clinical radiation therapy is the daily treatment set up of patient. Portal imaging can reduce the set up errors by verifying treatment plan, this need of improvement lead to the development of electronic portal imaging device (EPID) (11). In principle EPIDs convert x-rays into visible light by $\mathrm{Cu}$ / phosphors plate and the optical image is read out by the imaging system (20). In order to obtain high spatial resolution $\mathrm{Cu}$ /phosphors plate must be thin $(2 \mathrm{~mm})$, such a thin layer result in low x-ray absorption and thus smaller quantum efficiency (20), (22). Most of the EPID panels are made up of high atomic number $(Z)$ materials (20), that give an over-response to low energy x-rays which is undesirable for the dosimetric verifications (20), (19). In order to address these issues with EPID, Pang et al (20) for the first time proposed the Cherenkov radiation based detector with low $Z$ material which can have high quantum efficiency and resolution. In this work, they proposed Cherenkov portal imaging device (CPID) based on optical fiber taper coupled directly with flat panel imager (20). Theoretical validation of CPID was also confirmed by Monte Carlo simulation on Geant4 toolkit (24). In this work, CPID is made up of fused silica fibers focused towards X-ray source and the gap between them is filled with absorber. They used 0.48 numerical aperture (NA) for simulating the effect, when MV x-rays interact with CPID through Compton effect charged particles are emitted and resulting in Cherenkov radiation in the core of fused silica.(24). The schematic of the simulation is presented in figure 1.9.

In one of the works Teymurazyan and Pang (25), used Monte-Carlo simulation to study the effect of scattering from patient on the signal and noise of CPID in comparison with EPID. They concluded that CPID detected $50 \%$ lesser scattered signal than conventional EPID and differential signal to noise 


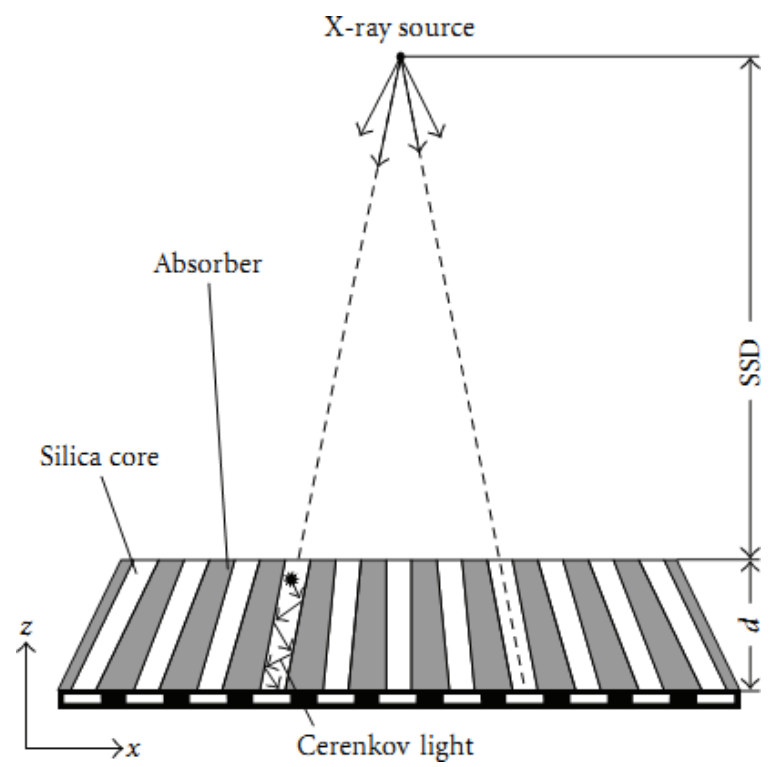

Figure 1.9: Schematic of CPID (Adapted from Teymurazyan and Pang (2012))

ratio from CPID has improved significantly. In another work, based on Monte-Carlo simulation, Pang et al (26) confirmed the feasibility of CPID with high density material such as lead-glass, whose quantum efficiency and DQE is much higher than EPIDs, moreover, the proposed CPID has spatial resolution similar to that of EPID. Silva and Pang (23) carried out an experimental study with single silica optical fiber, and evaluated different properties such as energy, angular dependance, and spectrum of Cherenkov radiation.

Cherenkov radiation has also been used to estimate the radiation dose to flat and curved surfaces, Pogue et al (29) has carried out both Monte-Carlo simulation and phantom studies. In 2013, Pogue group (9), has also proposed that intrinsic Cherenkov emission from medium can be imaged and used for spatially imparted dose distribution. In one of the works Glaser et al (8) has also employed Cherenkov radiation for estimating the dose delivered by IMRT and VMAT in water phantom using ICCD camera.

\subsection{Definition of Problem}

From the literature review it has been realized that Cherenkov radiation has potential for being applied for portal imaging. Most importantly it has been observed that Cherenkov radiation are not responsive to the scattered radiation, and there is a scope of studying and developing a prototype anti-scatter detector based on Cherenkov radiation. 


\subsubsection{Hypothesis}

The hypothesis is that with the anti-scatter detector we will be able to effectively reduce the detrimental effects of scattered radiation for megavoltage x-ray imaging.

\subsubsection{Specific objectives}

In the literature, it is observed that most of the work has been carried out with Cherenkov detectors made up of optical fiber, the fiber is made up of concentric core and cladding materials with slightly different refractive indices. In this work, we are proposing a $1 \mathrm{~mm}$ thin and $10 \mathrm{~cm}$ long glass rod as a detector whose cladding is air, in order to get maximum signal generated and propagating through it.

In this work, we will compare the response of glass rod based Cherenkov detector to x-ray scatters with that of ion chamber based detector. A practical large area detector will consist of many glass rods or ion-chambers, but in this work we mainly used single glass rod or single ion-chamber for measurements. In this work, we embedded both types of single pixel detector in glass slab to provide scatter conditions that a large area detector will create. 



\section{Chapter 2}

\section{Experimental Methodology}

In this chapter the procedure, material and methods of experimental work carried out in thesis are discussed in detail. Firstly experimental procedure carried out with ionization chamber are explained, followed by measurements of Cherenkov radiation with glass rod.

\subsection{Ionization Chamber}

For measuring the dose delivered by photon beam usage of ionization chambers has been recommended by the TG51 protocol (1). An ionization chamber is made up of a known material with a cavity of a known volume and it is used to measure the absorbed dose in a medium (15). It collects all the charge liberated $Q$ by the radiation in the cavity and this charge is measured accurately by electrometer. According to TG51 protocol, the absorbed dose $D_{w}^{Q}$ in the medium (water) under reference conditions is calculated by the following general formula (1):

$$
D_{w}^{Q}=M N_{D, w}^{Q}
$$

where $N_{D, w}^{Q}(\mathrm{~Gy} / \mathrm{C})$ is the absorbed dose water calibration factor for an ion chamber located in a beam, and $\mathrm{M}$ is the corrected electrometer reading in coulombs $(\mathrm{C})$. The electrometer reading is corrected for certain factors such as ion recombination, polarity, electrometer calibration effects, and environmental conditions of temperature and pressure (1) . Therefore, according to TG51 protocol (1) the corrected electrometer reading $\mathrm{M}$ is given by the following equation:

$$
M=P_{\text {ion }} P_{T P} P_{\text {elec }} P_{\text {pol }} M_{\text {raw }}(C)
$$

where $M_{\text {raw }}$ is the raw ion chamber reading in Coulombs $(\mathrm{C}) ; P_{T P}$ is the temperature-pressure correction for the standard environmental conditions; $P_{i o n}$ corrects for incomplete ion collection efficiency; $P_{\text {pol }}$ corrects for any polarity effects; and $P_{\text {elec }}$ considers the electrometer's calibration factor if electrometer and ionization chamber are calibrated separately. 
Since calibration factors are given for standard environmental conditions of temperature at $T_{0}=$ $22^{\circ} \mathrm{C}$ and pressure at $P_{0}=101.33 \mathrm{kPa}$ ( 1 atmosphere), $P_{T P}$ corrects charge to standard environmental conditions using following equation(1):

$$
P_{T P}=\frac{273.2+T}{273.2+22.0} \cdot \frac{101.33}{P},
$$

where $\mathrm{T}$ is the temperature in degrees Celsius at the ion chamber and $\mathrm{P}$ is the pressure in kilopascals.

Electrometers calibration factor $P_{\text {elec }}$ is considered 1.00 if the electrometer and ion chamber are calibrated as a unit and in Canada it is a common practice to calibrate them as a unit (1).

Other correction factors; $P_{i o n}$ for the pulsed beam and $P_{p o l}$ are calculated by the following formulas:

$$
P_{\text {ion }}\left(V_{H}\right)=\frac{1-V_{H} / V_{L}}{M_{\text {raw }}^{H} / M_{\text {raw }}^{L}-V_{H} / V_{L}}
$$

and

$$
P_{\text {pol }}=\left|\frac{\left(M_{\text {raw }}^{+}-M_{\text {raw }}^{-}\right)}{2 M_{\text {raw }}}\right|
$$

These correction factors remain constant for ionization chamber and electrometer, these factors remain constant during our measurements, thus they were measured only once and included in the final measurement whereas temperature-pressure correction factor was calculated for every set of measurement.

\subsubsection{Experimental Setup}

In the present study, we are carrying out all measurements with $6 \mathrm{MV}$ photon beam. A $30 \times 30 \mathrm{~cm}$ solid water phantom is placed at isocenter and ionization chamber is placed at different air gaps i.e. 10, 30 and $46 \mathrm{~cm}$ away from the solid water. The solid water phantom is made up of special resin material which has scattering and attenuating properties for x-rays quite similar to that of water. Solid water is available in the form of slabs of different dimensions. The field size was changed from $3 \times 3$ to $20 \times 20 \mathrm{~cm}^{2}$, and $300 \mathrm{MUs}$ were delivered every time at the machine output rate of $600 \mathrm{MU} / \mathrm{min}$. $\mathrm{MU}$ is monitoring unit, output of ion-chamber installed in LINAC head calibrated as; $1 \mathrm{MU}=1 \mathrm{cGy}$ dose delivered at isocentre under standard calibration conditions. The rationale behind changing the field size is to measure the scatter from the head of the LINAC. These measurements were also repeated without solid water phantom in order to find the scattering due to phantom. For all the measurements, ionization chamber was placed in the cylinder made up of $1.5 \mathrm{~cm}$ black delrin material which is acting as a build up material. The schematic is shown in fig 2.1.

The results for ionization chamber were also repeated by placing ionization chamber in the glass slab of dimensions of $30 \times 30 \times 10 \mathrm{~cm}^{3}$. This experiment is performed to study the scatter contribution from large area detector.

The obtained results are presented in the chapter 3. The results from the ionization chamber are recorded for comparison with the trend for Cherenkov radiation emitted by glass rod. The experimental 


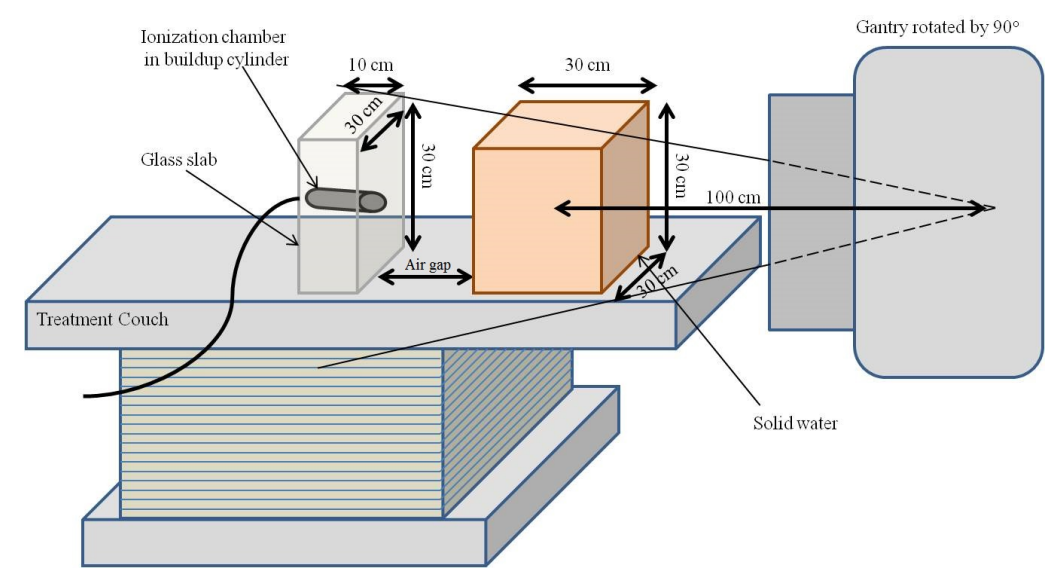

Figure 2.1: Schematic of measurement with ionization chamber in glass slab

set up for detection of Cherenkov radiation are presented in the following sections.

\subsection{Cherenkov radiation setup}

The same electrometer that was used for ion chamber readout was used to measure Cherenkov radiation as well. For measuring Cherenkov radiation the above mentioned setup was modified on the basis of theory of creation of Cherenkov radiation. When x-rays pass through dielectric material and as a result of Compton effect electrons are created and these fast moving electrons create Cherenkov radiation. In these experimental setups glass rod was used to create Cherenkov radiation. The experimental details are explained in the following subsections.

\subsubsection{Single Glass rod}

The glass rod with diameter $1 \mathrm{~mm}$ and $10 \mathrm{~cm}$ length was used to create Cherenkov radiation, this glass rod was placed in custom designed cylinder made up of black delrin and this cylinder is acting as buildup material of $1.5 \mathrm{~cm}$. After placing the rod in delrin cylinder, the cylinder was placed in the glass slab of dimensions $30 \times 30 \times 10 \mathrm{~cm}^{3}$ which was kept at different air gaps such as 10,30 and $46 \mathrm{~cm}$. It is exactly the same experimental set up followed in section 2.1.1

When $6 \mathrm{MV}$ x-ray beam from LINAC passes through the glass rod, as a result of Compton effect, fast moving electrons are created and these electrons when traverse through dielectric material create Cherenkov radiation. The Cherenkov signal coming out of glass rod was fed to Hamamatsu R6095 photomultiplier tube (PMT), placed outside the LINAC bunker, through $10 \mathrm{~m}$ long optical fiber with core diameter of $700 \mu \mathrm{m}$ and SMA adapter. The glass rod is coupled with optical fiber by using custom bare fiber SMA adapter of Bullet make as shown in figure 2.2. The glass rod in SMA adapter is fitted in custom designed delrin cap having SMA-SMA connector, on other end of this connector optical fiber 
is plugged in. In between the ends of glass rod and optical fiber, a small quantity of optical silicone gel is added so that light coming out of glass rod does not scatter while also acting as a refractive index matching gel. Another important factor is the polishing of end of glass rod which is to be coupled with optical fiber, polishing not only cleans the scratches on the end of glass surface, but also reduces the optical losses at the interface between glass rod and optical fiber.

Polishing plays a vital role. It was carried out with polishing papers bought from Thor Labs and the

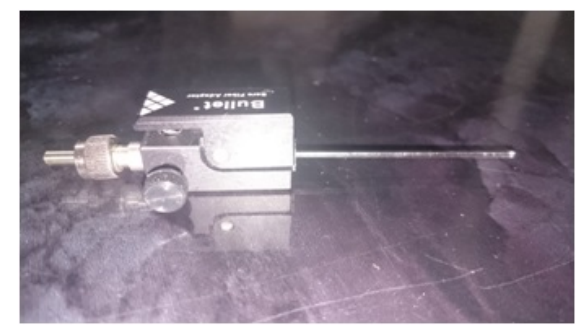

Figure 2.2: Polished glass rod in the bare fiber adapter

comparative images of polished and unpolished rod end are presented in figure 2.3. Polishing improved the reproducibility of signal drastically and results of polished and unpolished rod are discussed in the following chapter.

The measurement were taken in exactly similar way as described for ionization chamber. The x-ray beam of $6 \mathrm{MV}$ was irradiated at the rate of $600 \mathrm{MU} / \mathrm{min}$ and $300 \mathrm{MU}$ was delivered to the glass rod placed in buildup material in the glass slab. The field size was changed from $3 \times 3$ to $20 \times 20 \mathrm{~cm}^{2}$ in steps and air gap was set to 10, 30 and $46 \mathrm{~cm}$. These observations were carried out in the presence and absence of solid water phantom. All these readings are reported and discussed in chapter 3 .

\subsubsection{Data acquisition system}

The glass rod is coupled with one end of optical fiber whose other end is attached with PMT kept outside the LINAC bunker, Cherenkov photons striking at dynodes of PMT creates photoelectrons and these electrons are counted by electrometer of Vikotreen make with $0 \mathrm{~V}$ bias voltage. The Cherenkov signal

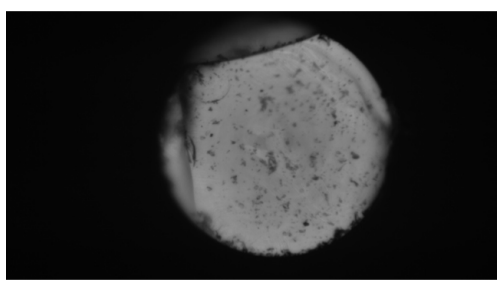

(a) Before polishing

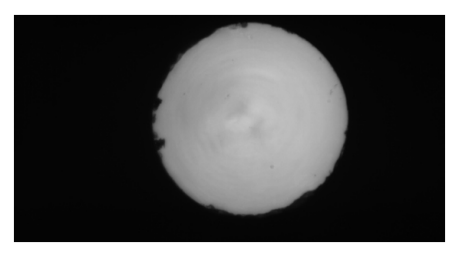

(b) After polishing

Figure 2.3: Effect of polishing on the surface of end of glass rod 
coming out from glass rod enters into optical fiber and the signal through fiber reaches the PMT and then light is being converted into charge which is measured by electrometer.

The setup is demonstrated in figure 2.4. The entire length of optical fiber present inside LINAC bunker can generate Cherenkov radiation but we are only concerned about the Cherenkov signal created by the glass rod. Therefore, correcting for this signal, a black paper is inserted in the coupling between glass rod and optical fiber, this blocked the Cherenkov radiation created by the rod from entering into fiber. Hence the Cherenkov radiation created only by optical fiber is read by electrometer and these readings act as a background. Then similar measurements were repeated after removing the black paper from the coupling, the signal is detected by electrometer. After subtracting the background signal from the signal, Cherenkov radiation generated by glass rod is obtained.

All these measurements were carried out twice and average of them were taken for calculating Cherenkov signal emanated by glass rod. Moreover, all these experiments on three different days to calculate the standard deviation and standard error and to confirm the reproducibility of the measurements.

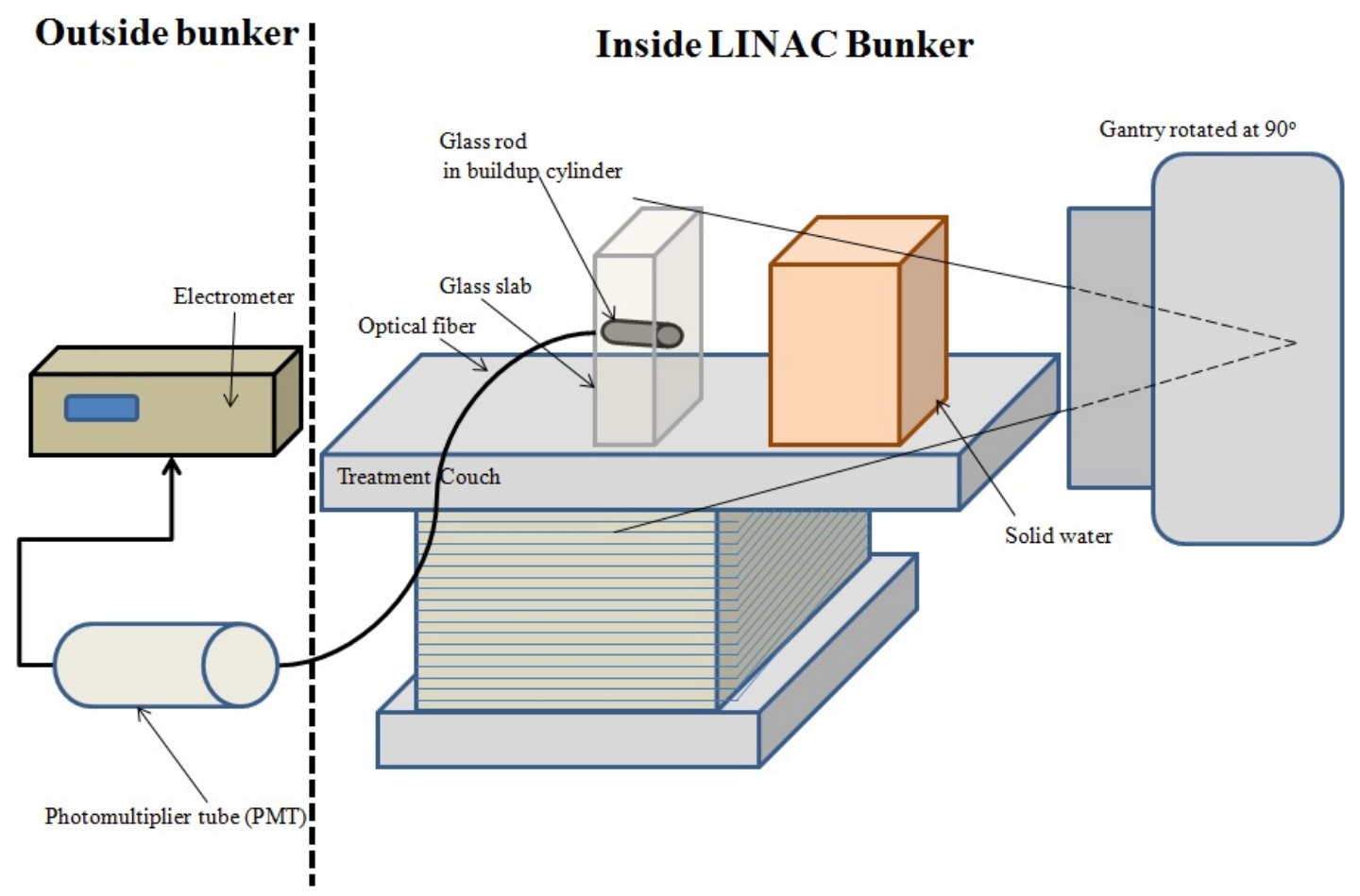

Figure 2.4: Schematic of experimental setup with data acquisition 


\subsubsection{Cherenkov radiation from group of glass rods}

In this experiment, instead of a single glass rod, bundle of a few glass rods were tightly packed in black delrin cylinder which acts as a buildup material. For coupling the light coming out of glass rods with optical fiber, optical silicone gel was introduced in between rods and fiber. Optical gel not only matched the refractive index of glass rod and optical fiber, but also restrained the light from glass rod from going into air.

Experimental procedures described in the previous section, was followed and the results from this setup are discussed in next chapter. 


\section{Chapter 3}

\section{Results and Discussion}

The results obtained from the different experimental setups explained in previous chapter are presented and discussed in this chapter.

\subsection{Results of Ionization Chamber}

\subsubsection{Ionization Chamber placed in air}

The charge created in ionization chamber when it was placed at different air gaps with different field sizes, was recorded by the electrometer. The recorded charge $(\mathrm{nC})$ is plotted as a function of field size at different air gaps. The signal measured by the electrometer is comprised of both primary and scatter components of the beam. In order to separate the total signal into primary and scatter components, method suggested by Jaffray et al (12) was adopted. The plotted data was extrapolated to zero field size, which implies that scatter component has reduced to zero and only the primary component of the beam is present. For extrapolation of the data, the curve was fitted by using cubic smooth and spline function in MATLAB, then the curve was extrapolated using MATLAB function (Sample code presented in Appendix 1).

The figure 3.1 to 3.3 shows the electrometer signal variation as a function of field size in the presence and absence of the solid water phantom when ionization chamber was placed at air gap 10, 30 and 46 $\mathrm{cm}$ respectively. All these measurements were carried out with ionization chamber kept in the buildup cylinder and placed in the air. Moreover, the plotted readings have also been corrected for temperature and pressure on the days of measurements.

From the figures 3.1 to 3.3 , it can be inferred that with the increase in field size, the total signal recorded by the ion chamber increases. In the present study, the signal from divergent beam of $6 \mathrm{MV}$ was measured, it is well known that primary component of beam remains constant for all the field sizes (12). The increase in signal as a function of field sizes is attributed to the scatter component of the beam. Since x-ray beam suffers large amount of scattering in the machine head, with the increase in 


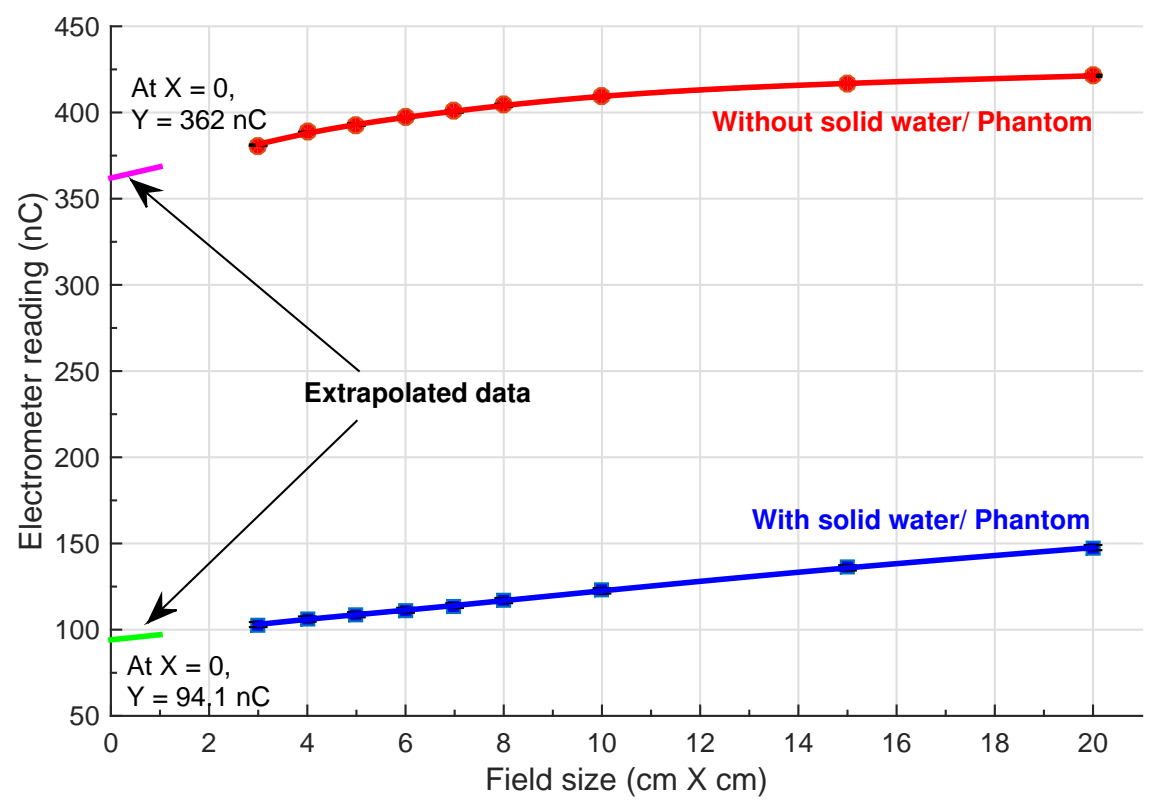

Figure 3.1: Electrometer readings by ionization chamber placed in air, at air gap $10 \mathrm{~cm}$

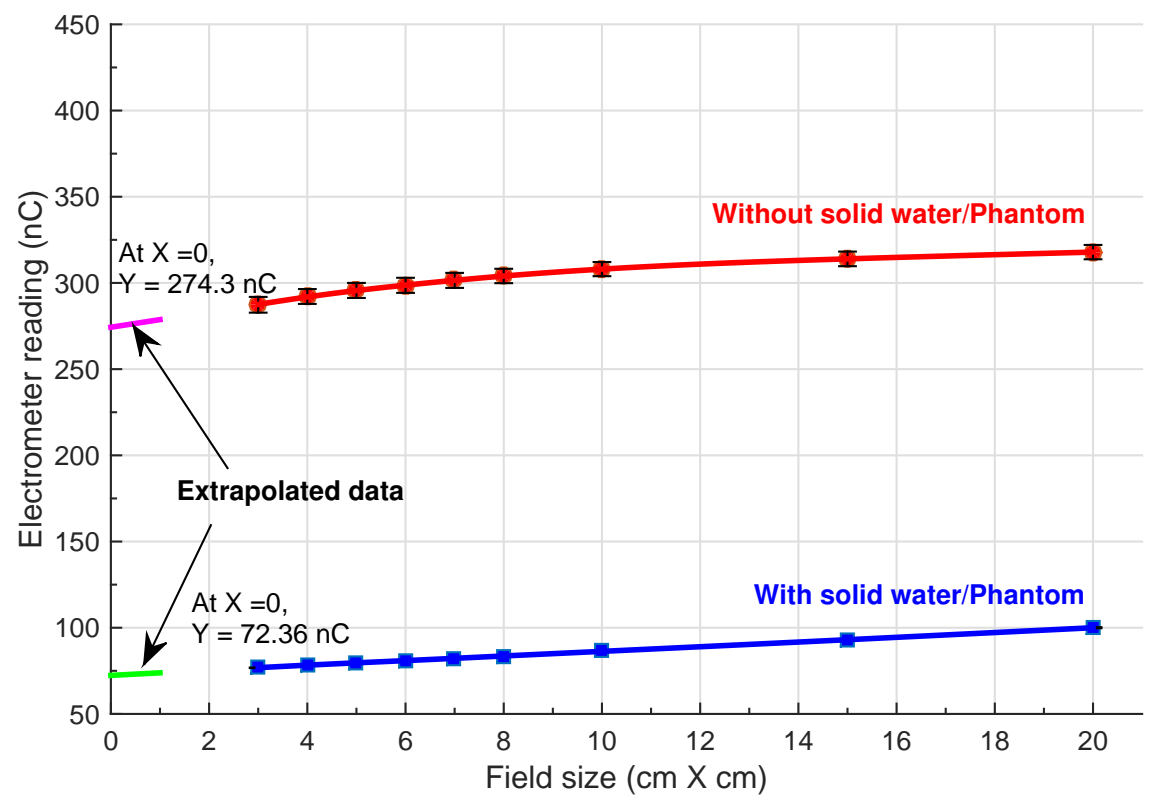

Figure 3.2: Electrometer readings by ionization chamber placed in air, at air gap $30 \mathrm{~cm}$ 


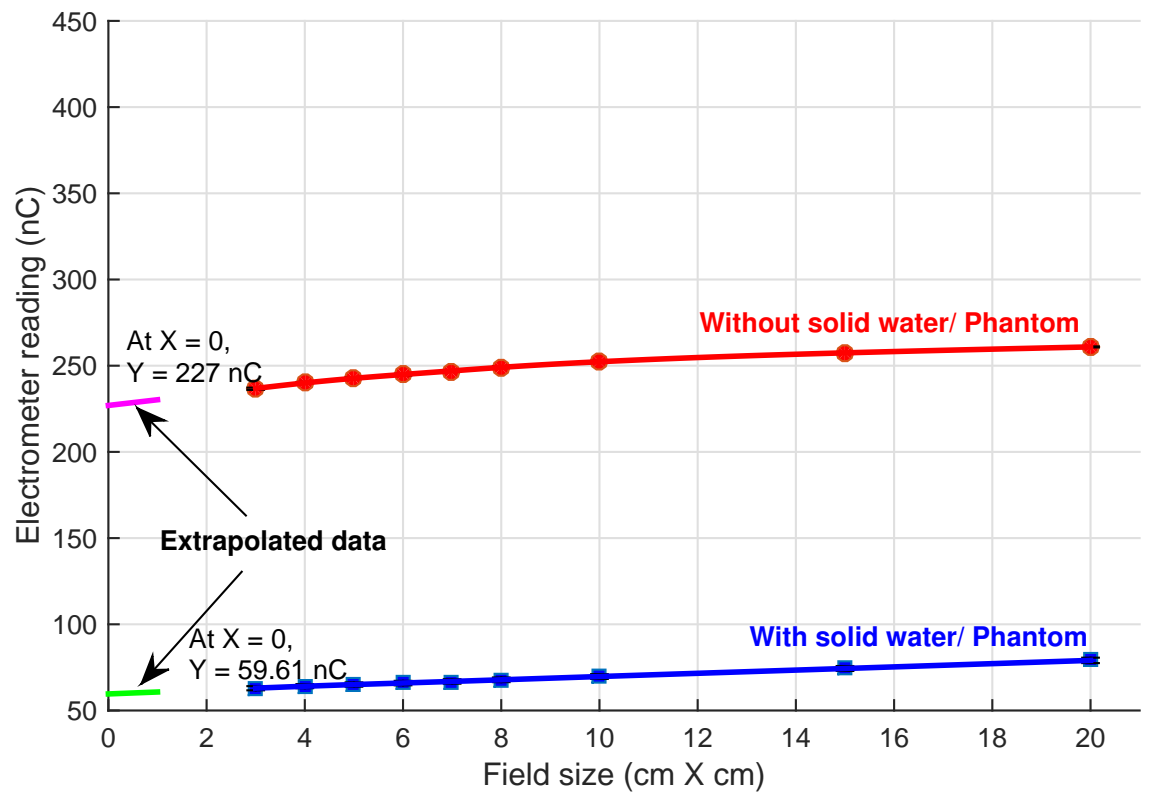

Figure 3.3: Electrometer readings by ionization chamber placed in air, at air gap $46 \mathrm{~cm}$

field size more scattered beam emanates from head, resulting in more signal.

In figures 3.1-3.3, it is observed that with the placement of solid water phantom at isocenter, total signal has decreased substantially. The primary beam transmitted through the slab and reaching the detector, has reduced significantly because of attenuation of the beam. The ratios of primary signal without and with the phantom for all the air gaps are calculated. The slab of $30 \mathrm{~cm}$ thick solid water attenuates the beam and this attenuation was calculated by the following formula:

$$
I=I_{0} e^{-\mu x}
$$

From the first principle calculations, the difference in theoretical and experimental signal is approximately 14 to $16 \%$.

Another observation that was made from figures 3.1-3.3, is the reduction in primary beam with air gap, which decreases according to inverse square law.

The scatter fraction (SF) was calculated by using formula suggested by Jaffray et al (12) at every field size and air gaps in the presence and absence of the phantom:

$$
S F=\frac{S}{S+P}=\frac{S}{T}
$$

where $\mathrm{S}$ is the scatter component, $\mathrm{P}$ is the primary portion of the beam and $\mathrm{T}$ is the total signal read by the ionization chamber. The calculated SF is plotted against the field size for different air gaps and 
they are presented in the figures 3.4 to 3.6 .

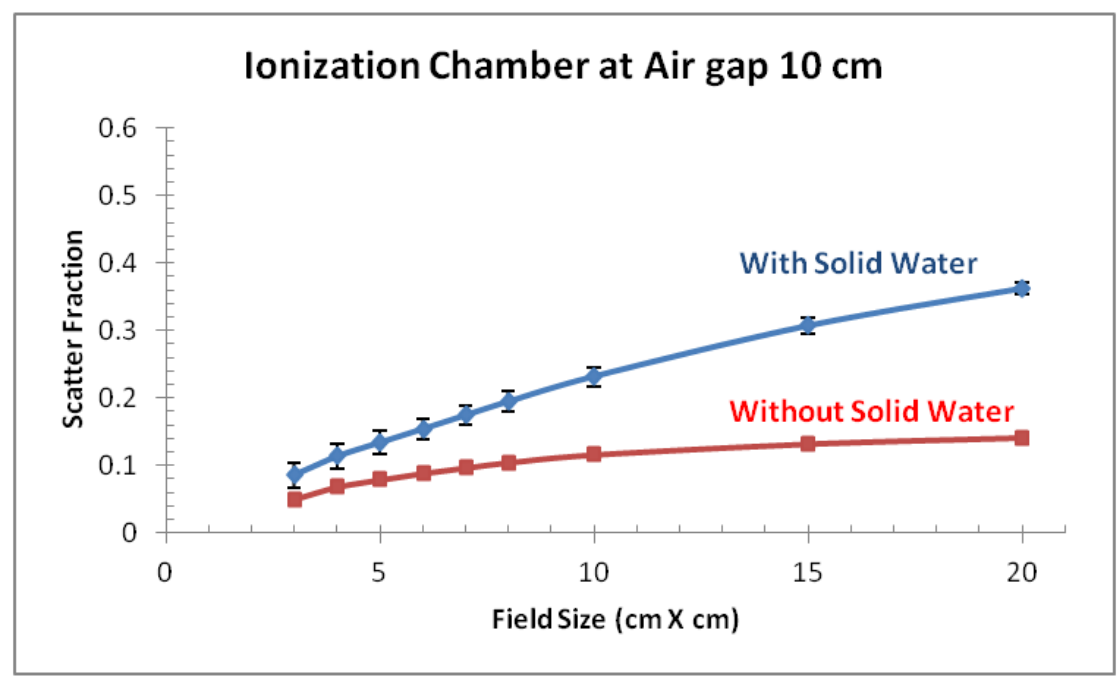

Figure 3.4: Scatter fraction by ion-chamber placed in air, at air gap $10 \mathrm{~cm}$

Figures 3.4-3.6 suggest that the scatter fraction in presence of solid water is higher than that without solid water, the reason is obvious that solid water has scattered the primary beam quite significantly. Another point that can be highlighted from these figures is that with the increase in field size the scatter fraction increases even without phantom, this increase in scatter component can be attributed to the scattering of beam from machine head. The air gap also effects the signal, since with air gap intensity of primary beam gets weaken and it also reduces the scatter component reaching the detector as well.

\subsubsection{Ionization Chamber in Glass slab}

In the last section, results of the ionization chamber placed in air were discussed. To study the scatter due to large area detector itself, an ionization chamber was placed in the glass slab of $30 \times 30 \times 10 \mathrm{~cm}^{3}$ as shown in the figure 2.1. The results obtained from this experimental setup are presented and discussed in the following section. Figures 3.7 to 3.9 represent the results obtained when the ionization chamber is kept at different air gaps namely 10,30 and $46 \mathrm{~cm}$.

The primary signal extrapolated from these curves are represented in Table 3.1, and they are also compared with the primary signal extrapolated when the chamber is placed in air.

From Table 3.1, it can be inferred that primary signal is almost same whether ionization chamber 


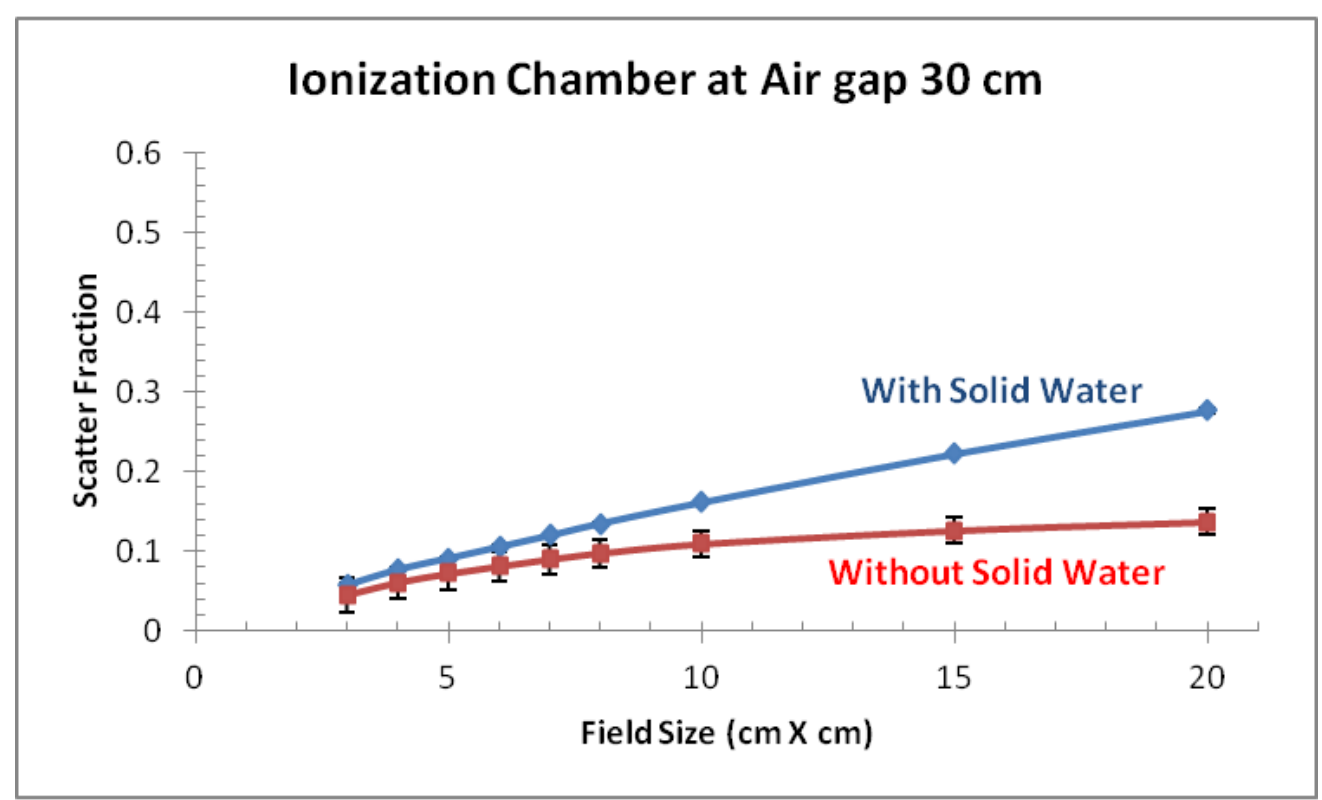

Figure 3.5: Scatter fraction by ion-chamber placed in air, at air gap $30 \mathrm{~cm}$

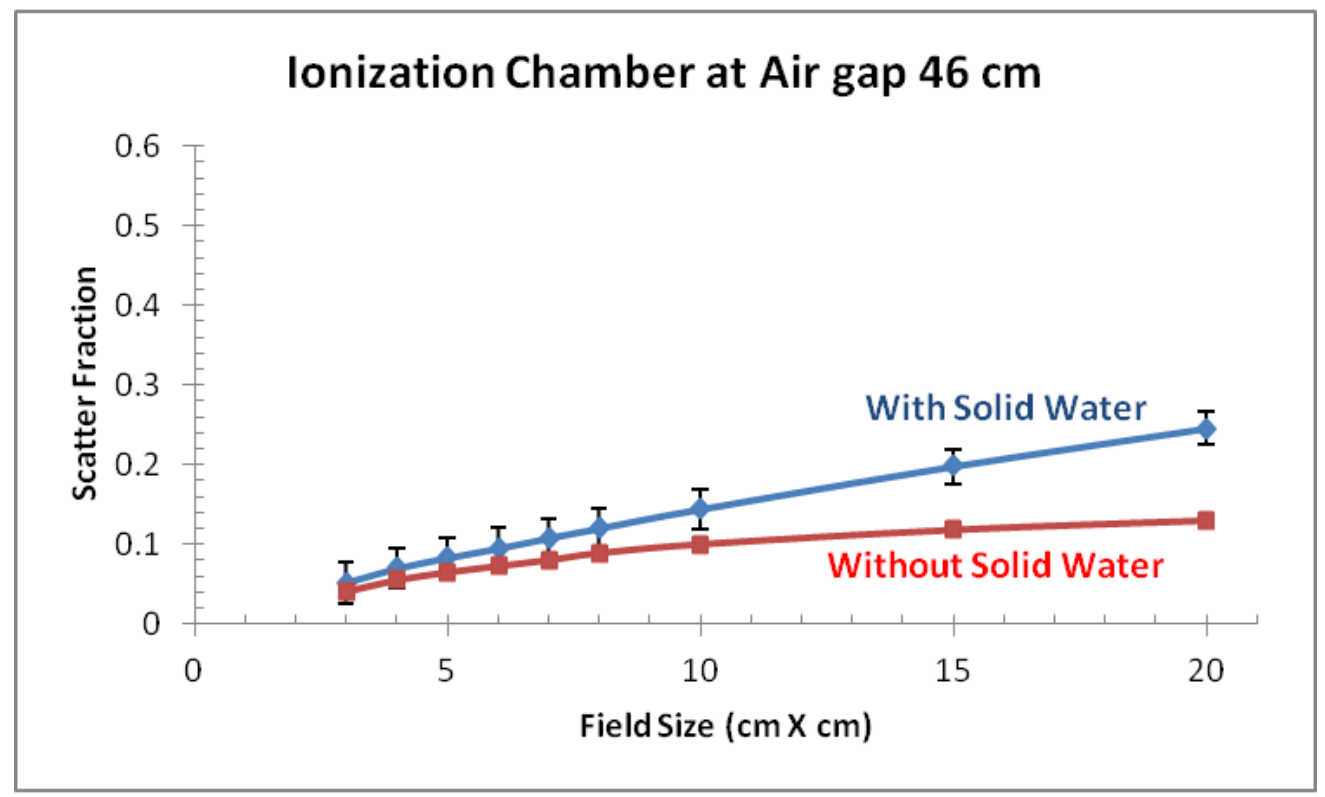

Figure 3.6: Scatter fraction by ion-chamber placed in air, at air gap $46 \mathrm{~cm}$ 


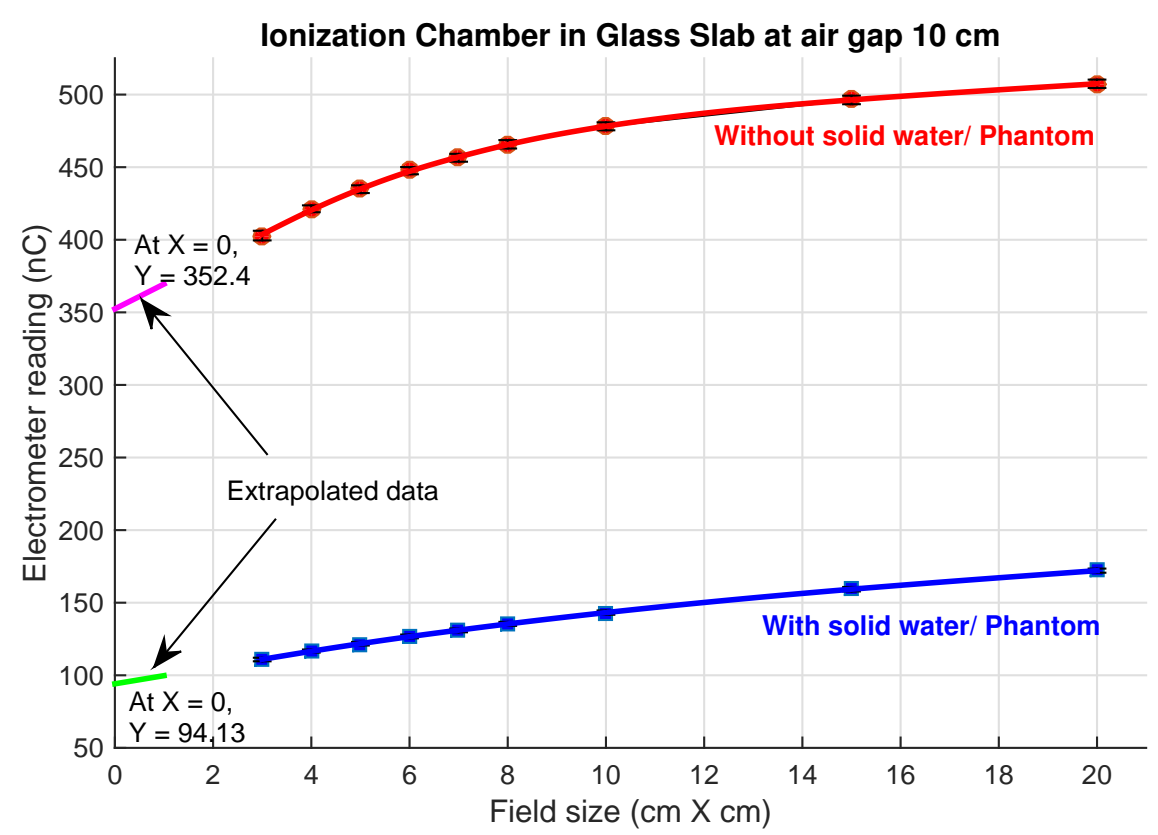

Figure 3.7: Electrometer readings by ion-chamber placed in glass slab, at air gap $10 \mathrm{~cm}$

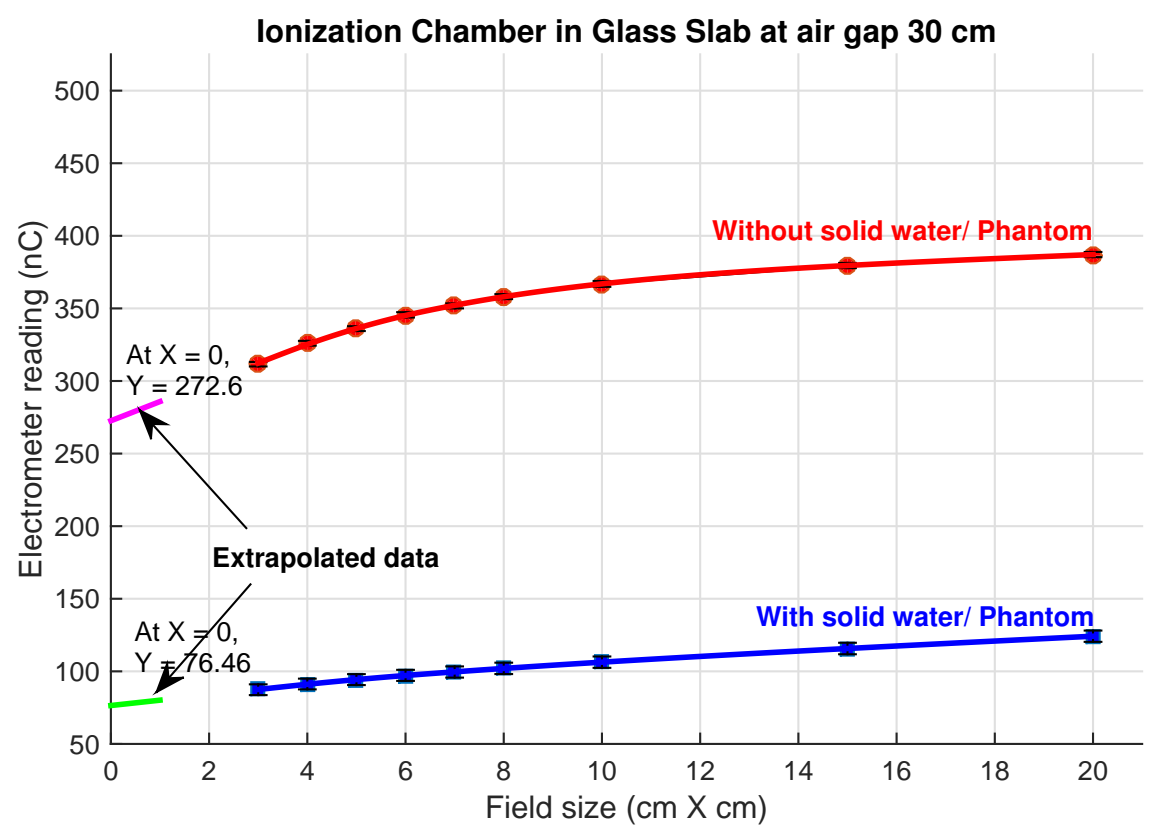

Figure 3.8: Electrometer readings by ion-chamber placed in glass slab, at air gap $30 \mathrm{~cm}$ 


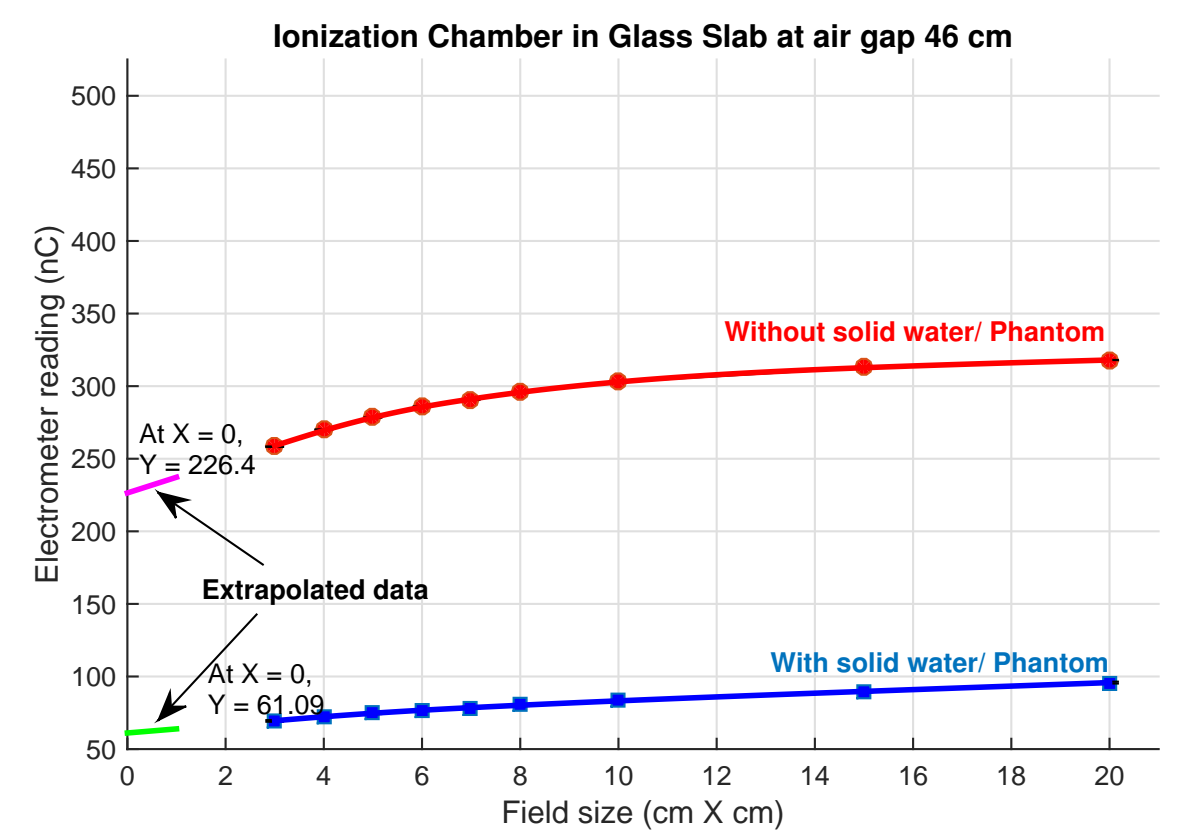

Figure 3.9: Electrometer readings by ion-chamber placed in glass slab, at air gap $46 \mathrm{~cm}$

\begin{tabular}{|c|cc|cc|}
\hline & Without solid water & & With solid water & \\
Air gap $(\mathrm{cm})$ & In air $(\mathrm{nC})$ & In glass slab $(\mathrm{nC})$ & In air $(\mathrm{nC})$ & In glass slab $(\mathrm{nC})$ \\
\hline \hline 10 & 362 & 352.4 & 94.1 & 94.13 \\
30 & 274.3 & 272.6 & 72.36 & 76.46 \\
46 & 227 & 226.4 & 59.61 & 61.09 \\
\hline \hline
\end{tabular}

Table 3.1: Primary signal obtained after extrapolated the curves represented in figures 3.1 to 3.3 and 3.7 to 3.9 


\begin{tabular}{|c|c|cc|cc|}
\hline $\begin{array}{c}\text { Field Size } \\
(\mathrm{cm} \times \mathrm{cm})\end{array}$ & $\begin{array}{c}\text { Air gap } \\
(\mathrm{cm})\end{array}$ & $\begin{array}{c}\text { Without solid water } \\
\text { In air }\end{array}$ & $\begin{array}{c}\text { In glass slab } \\
(\mathrm{nC})\end{array}$ & $\begin{array}{c}\text { With solid water } \\
\text { In air } \\
(\mathrm{nC})\end{array}$ & $\begin{array}{c}\text { In glass slab } \\
(\mathrm{nC})\end{array}$ \\
\hline \hline \multirow{3}{*}{3} & 10 & 381.1 & 402.9 & 102.9 & 110.9 \\
& 30 & 287.3 & 311.6 & 76.8 & 87.3 \\
& 46 & 236.6 & 258.3 & 62.9 & 69.4 \\
\hline \multirow{3}{*}{20} & 10 & 421.3 & 507.4 & 147.6 & 172.1 \\
& 30 & 317.9 & 387.1 & 100 & 124.2 \\
& 46 & 260.9 & 318 & 79.1 & 95.8 \\
\hline \hline
\end{tabular}

Table 3.2: Comparison of average total signal measured by ionization chamber when placed in air and glass slab

\begin{tabular}{|c|c|cc|cc|}
\hline $\begin{array}{c}\text { Field Size } \\
(\mathrm{cm} \times \mathrm{cm})\end{array}$ & $\begin{array}{c}\text { Air gap } \\
(\mathrm{cm})\end{array}$ & $\begin{array}{c}\text { Without solid water } \\
\text { In air }\end{array}$ & $\begin{array}{c}\text { In glass slab } \\
(\mathrm{nC})\end{array}$ & $\begin{array}{c}\frac{\text { With solid water }}{\text { In air }} \\
(\mathrm{nC})\end{array}$ & $\begin{array}{c}\text { In glass slab } \\
(\mathrm{nC})\end{array}$ \\
\hline \hline \multirow{3}{*}{3} & 10 & 0.050 & 0.125 & 0.086 & 0.150 \\
& 30 & 0.045 & 0.125 & 0.058 & 0.125 \\
& 46 & 0.041 & 0.124 & 0.053 & 0.120 \\
\hline \multirow{3}{*}{20} & 10 & 0.140 & 0.306 & 0.363 & 0.453 \\
& 30 & 0.137 & 0.295 & 0.276 & 0.394 \\
\hline \hline
\end{tabular}

Table 3.3: Comparison of scatter fraction measured by ionization chamber when placed in air and glass slab

is placed in air or in glass slab. Whereas, Table 3.2 compares the total signal for both the experimental setups, it is clear that total signal has increased substantially when ionization chamber was placed in glass slab. Since the primary signal remained almost the same for both setups, reason for increase in total signal may be attributed to the scatter of signal from glass slab. The contribution from the scatter by glass slab is almost from 7 to $20 \%$ of the signal at different field sizes and air gaps. The scatter fraction at different field sizes and air gaps are compared in the table 3.3, it is evident that for the same field size scatter fraction decreased with the increase in air gap. Moreover, with the placement of solid water at isocenter, scatter fraction increases, because solid water or phantom is one of the major source of scattering. Another point that can be highlighted from the data is; when chamber is placed in glass slab the scatter fraction increased, these findings confirm that the increase in the signal is due to scatter component added by glass slab. It can be inferred that for small field sizes the scatter fraction for the phantom and without phantom are very close and with the increase in field size scatter fraction for the solid water increases and is higher than that of without solid water. 


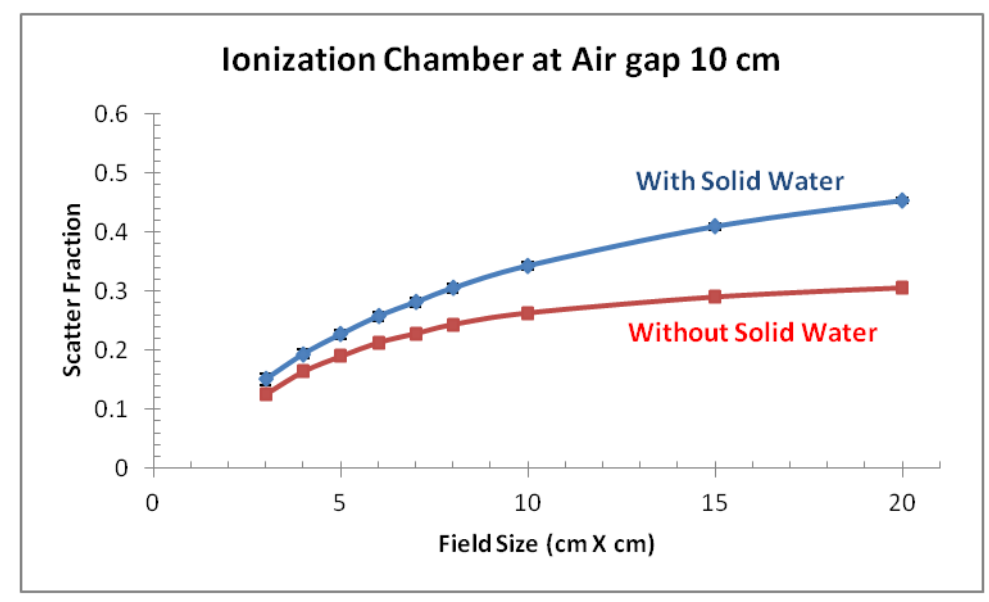

Figure 3.10: Scatter fraction by ion-chamber placed in glass slab, at air gap $10 \mathrm{~cm}$

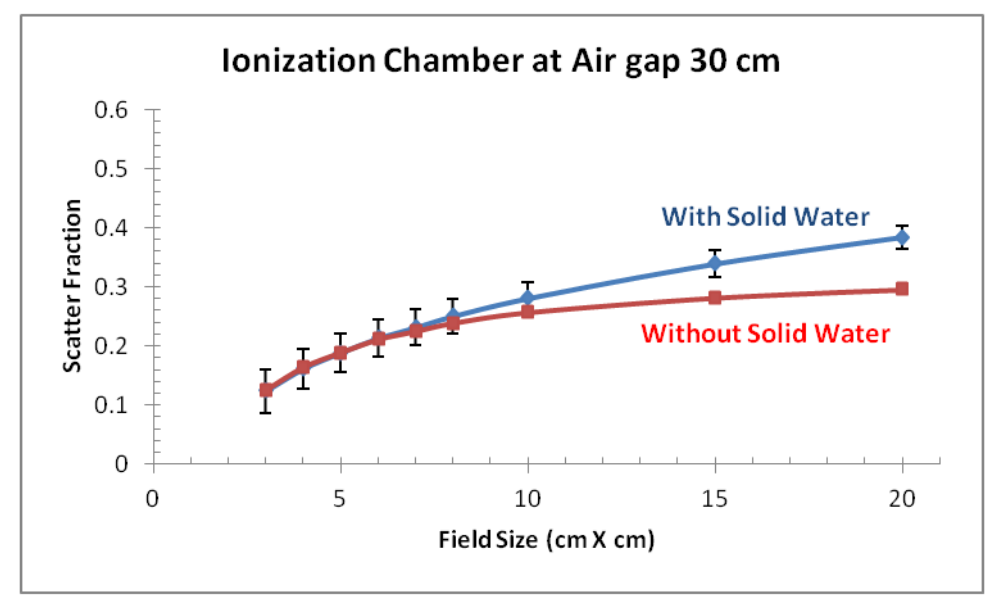

Figure 3.11: Scatter fraction by ion-chamber placed in glass slab, at air gap $30 \mathrm{~cm}$ 


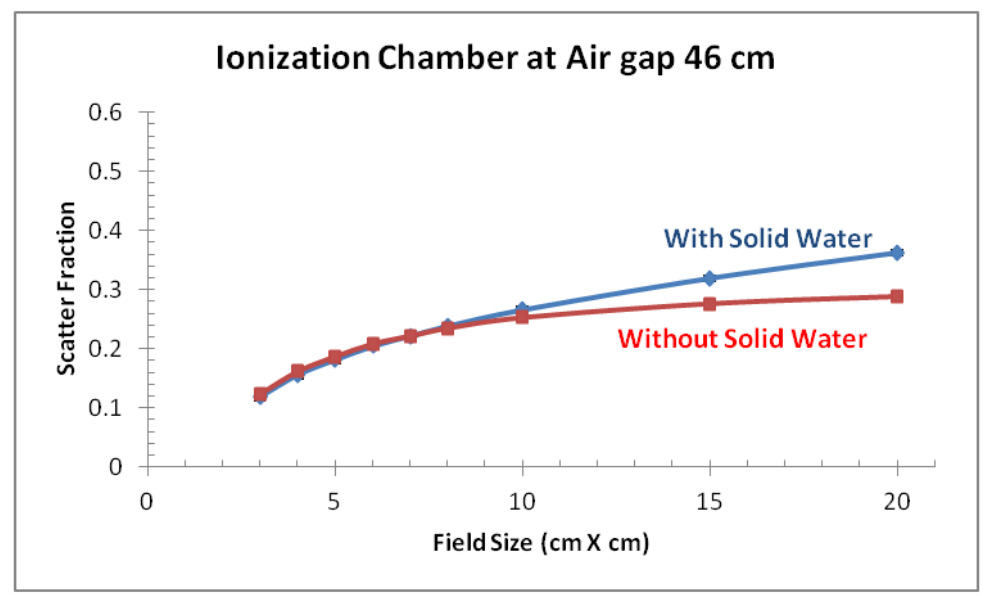

Figure 3.12: Scatter fraction by ion-chamber placed in glass slab, at air gap $46 \mathrm{~cm}$

\subsection{Results of Cherenkov Radiation}

As discussed in Chapter 2, different types of experimental setups were used to study Cherenkov radiation, in this section the results obtained when different types of dielectric materials are placed in $6 \mathrm{MV}$ x-ray beam, are presented and discussed in this section. All these readings were repeated on 3 different days to find out standard deviation and standard error from the mean of three observations.

\subsubsection{Effect of polishing on Cherenkov signal}

In chapter 2, the effect of polishing on the surface of glass rod has been shown in the figure 2.3, this effect is also evident on the results of Cherenkov signal. The unpolished glass rod was subjected to $6 \mathrm{MV}$ x-ray beam and Cherenkov signal was recorded in exactly same manner as explained in previous section. The results of unpolished rod is presented in fig 3.13. The results clearly indicate that unpolished rod has much higher standard error than that of polished rod, this makes the result highly uncertain. Therefore, polishing of the end of glass rod improves the interface and reduces the scattering of the Cherenkov signal from the end of glass rod.

All other measurements were carried out with polished glass rods only.

\subsubsection{Single glass rod}

When single glass rod is placed in x-ray beam of $6 \mathrm{MV}$, Cherenkov radiation is created and after passing through the optical fiber, PMT converts it into charge which is measured by electrometer. The results 


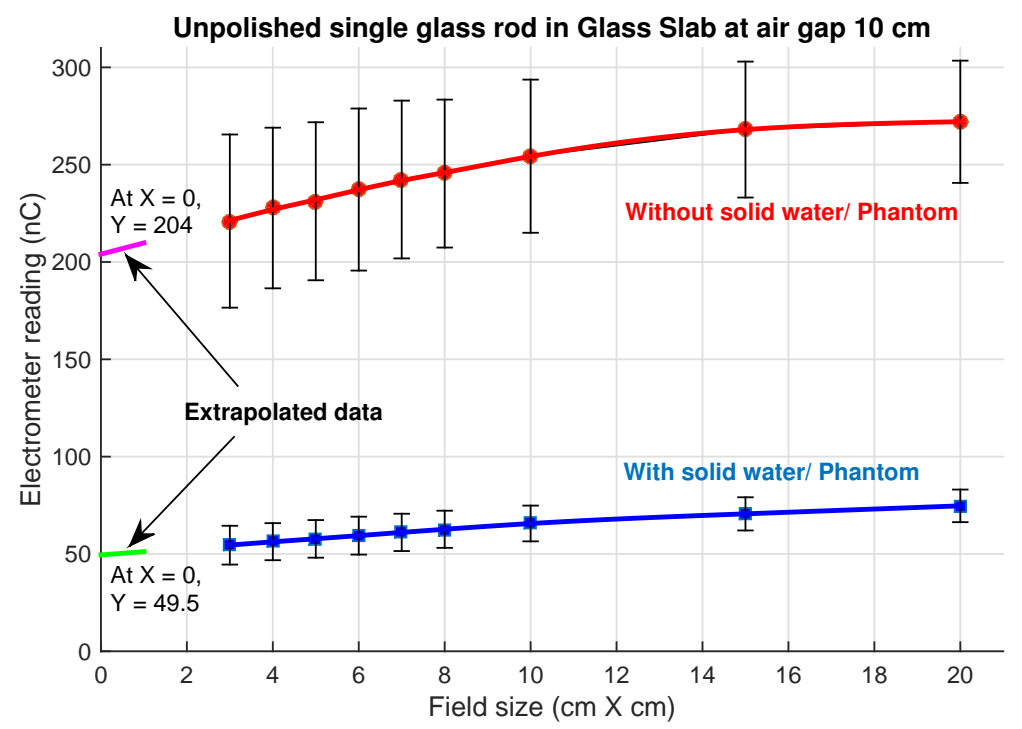

Figure 3.13: Cherenkov signal by unpolished rod placed in glass slab, at air gap $10 \mathrm{~cm}$

\begin{tabular}{|c|c|c|}
\hline Air gap $(\mathrm{cm})$ & Without solid water & With solid water \\
\hline \hline 10 & 174 & 49.66 \\
30 & 88.32 & 22.91 \\
46 & 40.83 & 11.51 \\
\hline \hline
\end{tabular}

Table 3.4: Primary signal from Cherenkov radiation obtained after extrapolated the curves

obtained for Cherenkov radiation from polished single rod at air gap of $10 \mathrm{~cm}$ for different field sizes are presented in figure 3.14. The results indicate that with increase in field size the Cherenkov signal increased, similar trend was observed by measurements by ionization chamber. The primary signal was obtained by extrapolating the recorded data, at field size $20 \times 20 \mathrm{~cm}^{2}$ the signal increased by $46 \%$ from the primary signal. Another point that can be noted is that with the placement of solid water, the signal decreased substantially because of attenuation. In these results, standard deviation is much higher than that observed in the results by ionization chamber. The reasons of uncertainty may be attributed to the design of coupling between glass rod and optical fiber, another reason may be the signal measurement setup of PMT and electrometer.

In the next experiment the glass rod was moved to other air gaps such as $30 \mathrm{~cm}$ and $46 \mathrm{~cm}$, the results are presented in the figures 3.15 and 3.16 respectively. From the results it can be confirmed that with the increase in air gap the signal decreased, whereas with the increase in field size signal increases. From these results, primary component of Cherenkov signal was found by extrapolating the data to field size $0 \times 0 \mathrm{~cm}^{2}$, by using the MATLAB program shown in Appendix 1. The primary signals at different air gaps are summarized in table 3.4. These results signify that with the increase in air gap, primary signal 


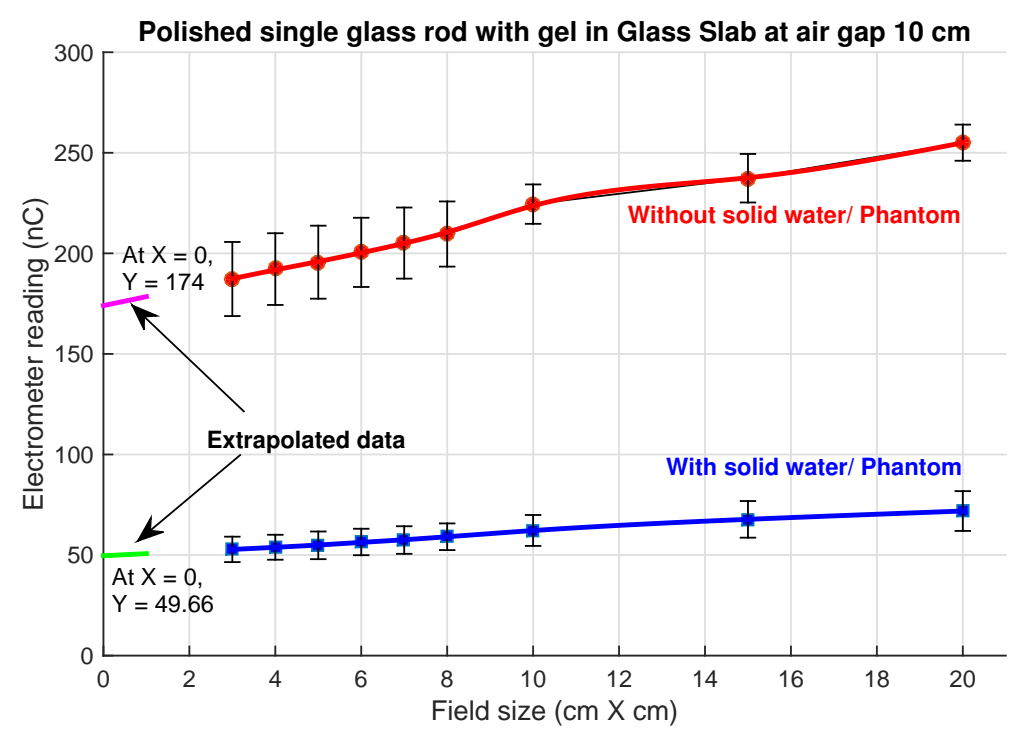

Figure 3.14: Cherenkov signal by polished glass rod placed in glass slab, at air gap $10 \mathrm{~cm}$

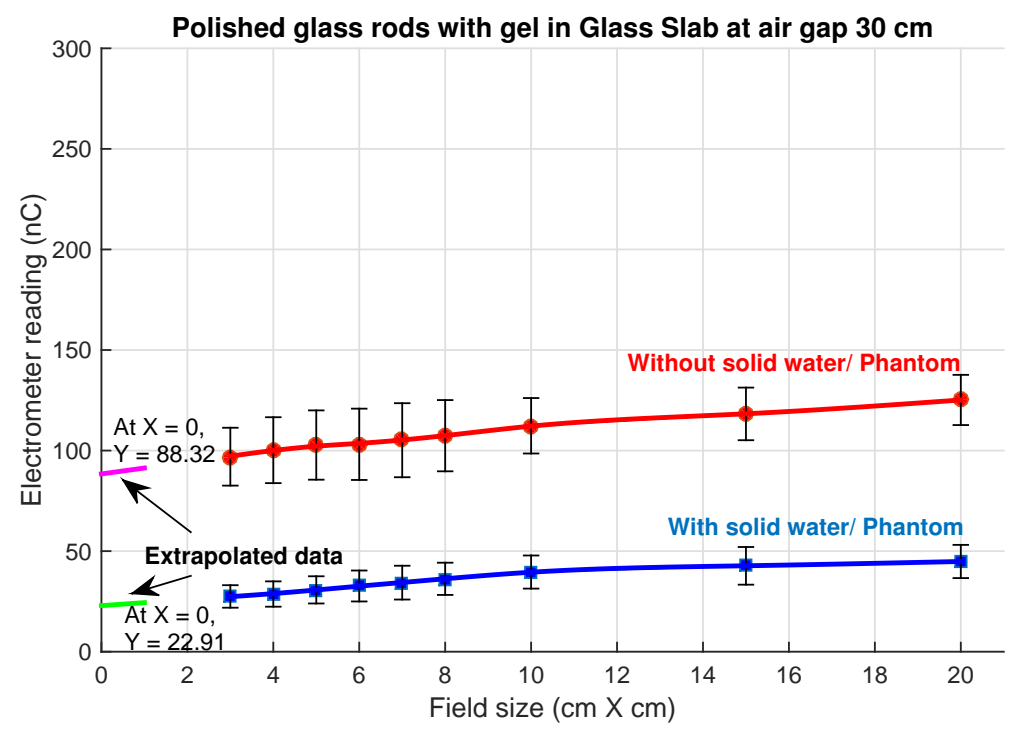

Figure 3.15: Cherenkov signal by polished rod placed in glass slab, at air gap $30 \mathrm{~cm}$ 


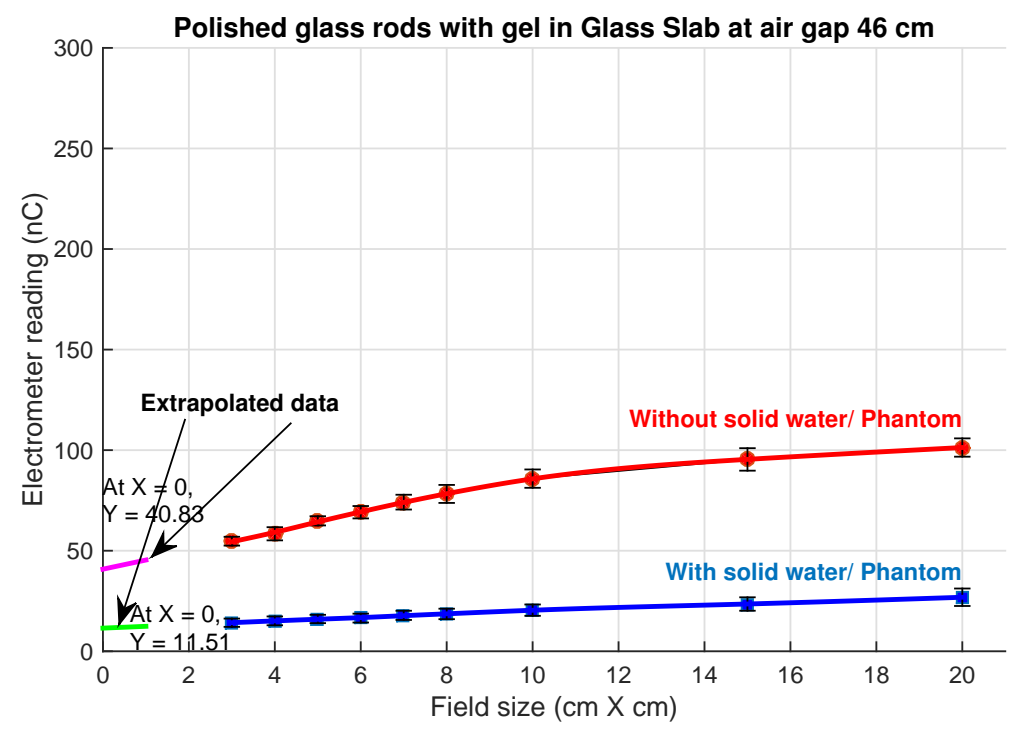

Figure 3.16: Cherenkov signal by polished rod placed in glass slab, at air gap $46 \mathrm{~cm}$

has weakened a lot and at air gap of $46 \mathrm{~cm}$ in presence of solid water the Cherenkov signal is merely detectable. Moreover, as anticipated with the placement of solid water the signal reduces substantially due to attenuation of signal by phantom.

The signal was analyzed to calculate scatter fraction, scatter fraction versus field size for the different air gaps are represented in figures 3.17 to 3.19 . At air gap $10 \mathrm{~cm}$ the scatter fraction with phantom and without phantom are almost same and curves are overlapping, this clearly shows that Cherenkov based detector is not responding to the scatter component of beam from the phantom. If it is compared with the signal from ionization chamber, the scatter fraction from Cherenkov detector is less than that of ion-chamber (see figure 3.10).

At air gap $30 \mathrm{~cm}$, the scatter fraction for solid water is greater than without solid water, but if the uncertainties in the signal are taken into account it can be concluded that scatter fraction in both cases are overlapping. At air gap $46 \mathrm{~cm}$, Cherenkov signal has weakened drastically in the presence of phantom, therefore the calculation of scatter fraction may become erroneous because of very small signal and moreover, the uncertainties calculated are overlapping in both cases.

\subsubsection{Group of glass rods}

Another experiment was designed to confirm the Cherenkov radiation was explained in section 2.2.3, in which group of glass rods were tightly packed in buildup cylinder and then they were irradiated with $300 \mathrm{MU}$ of $6 \mathrm{MV}$ x-ray beam. The coupling played a crucial role in making signal reproducible. In this case group of rods are coupled with single optical fiber, so it is important to make all the light emitted 


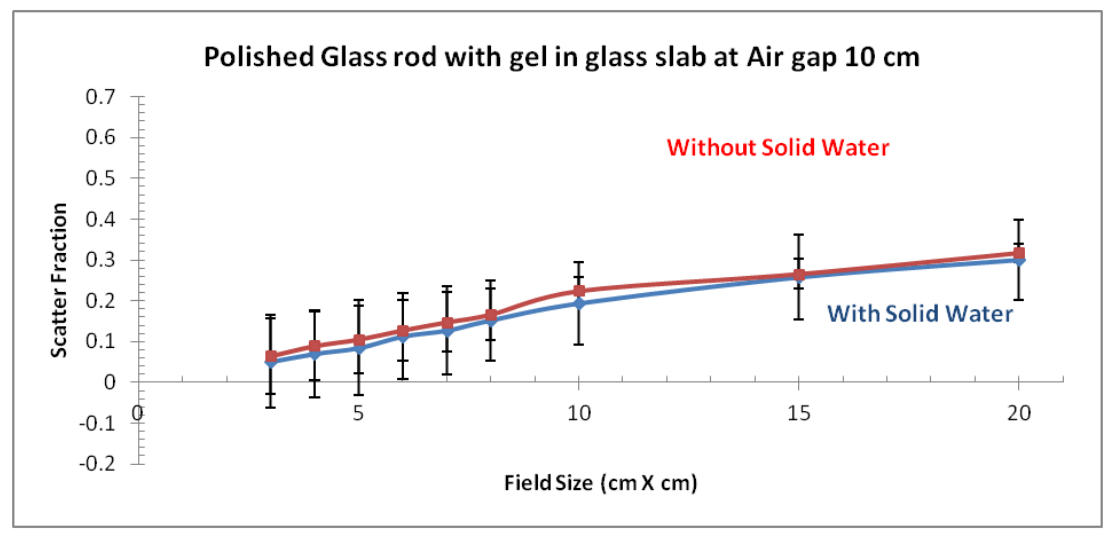

Figure 3.17: Scatter fraction by polished rod, at air gap $10 \mathrm{~cm}$ 


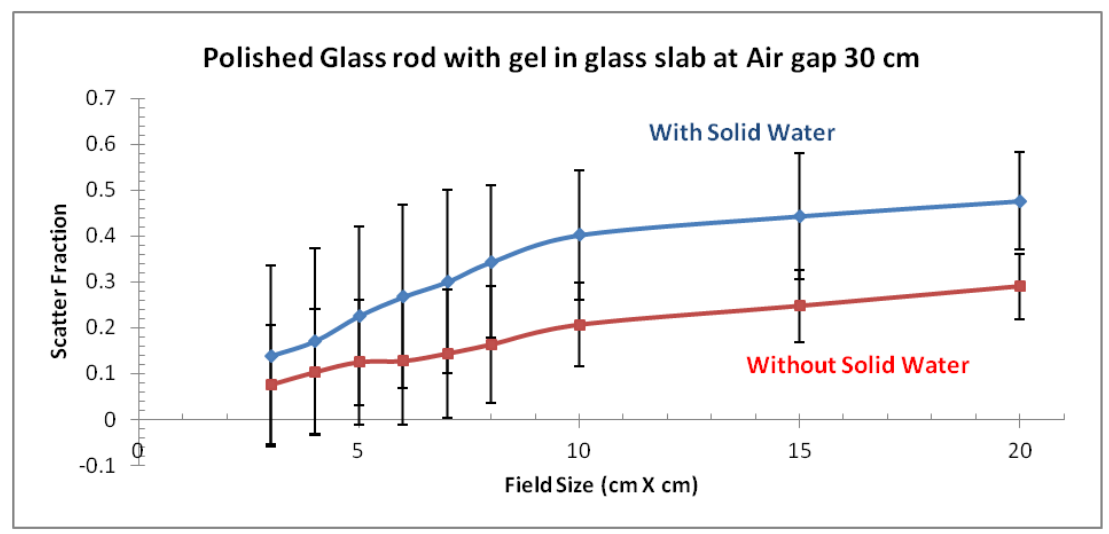

Figure 3.18: Scatter fraction by polished rod, at air gap $30 \mathrm{~cm}$ 


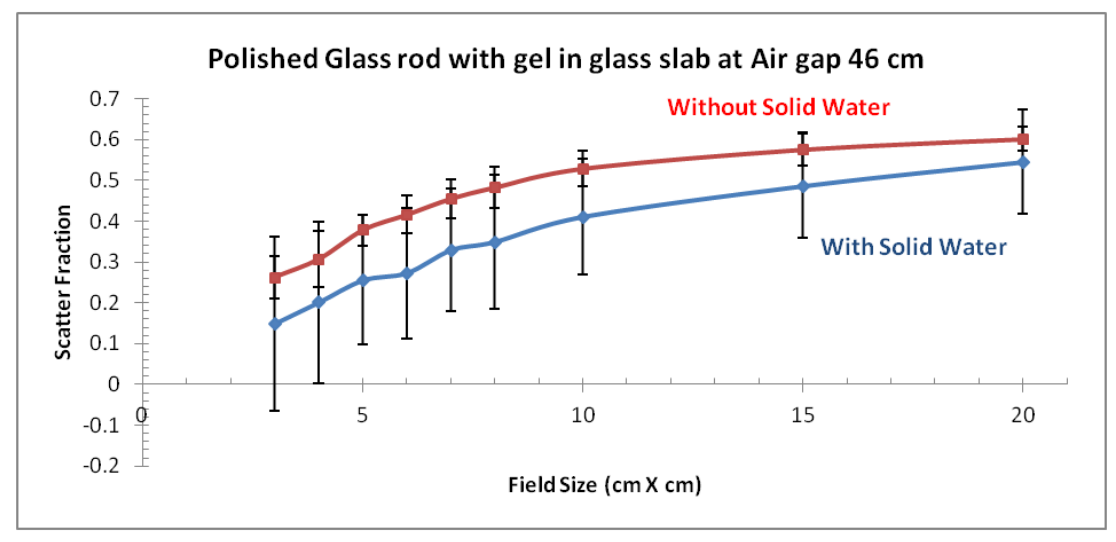

Figure 3.19: Scatter fraction by polished rod, at air gap $46 \mathrm{~cm}$ 
by rods enter into fiber. Since the diameter of optical fiber $(0.7 \mathrm{~mm})$ is much smaller than glass rod (1 $\mathrm{mm})$, therefore all created light will not enter the fiber. In order to make Cherenkov radiation created in rods to enter into fiber, an optical silicone gel was used as a coupling medium. On one side group of rods are penetrating and on other side optical fiber is collecting the signal. Cherenkov signal created by group of rods is measured by same method described in section 2.2. The results when group of rods were placed at air gaps 10, 30 and $46 \mathrm{~cm}$ for different field sizes are shown in figures 3.20 to 3.22 .

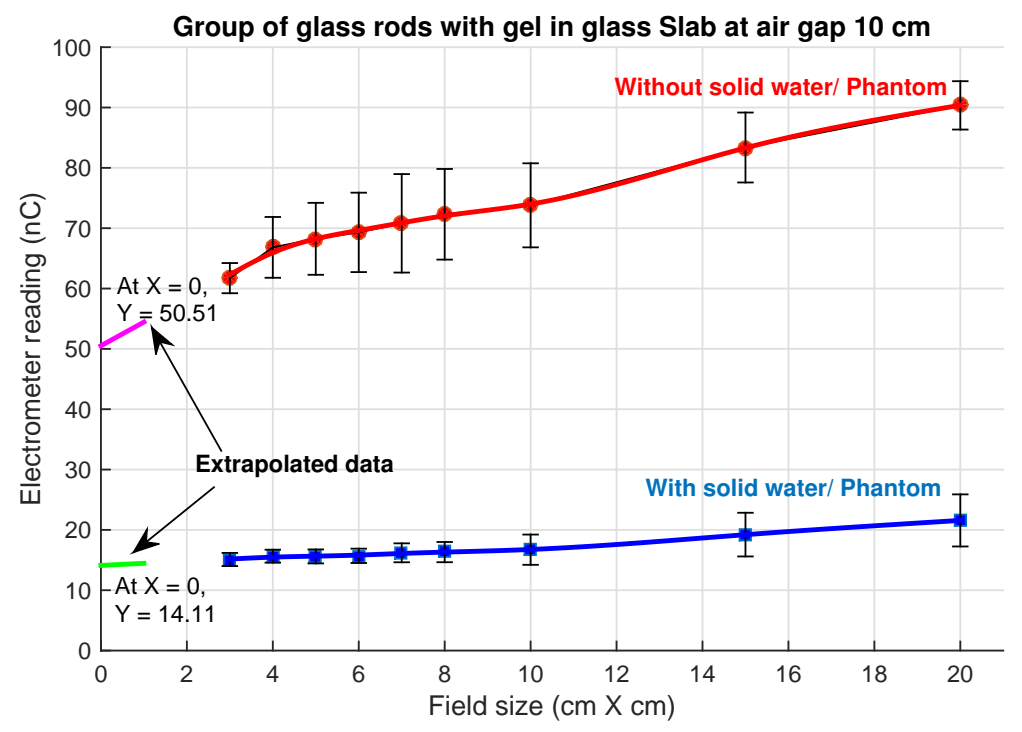

Figure 3.20: Cherenkov radiation from group of rods, at air gap $10 \mathrm{~cm}$

Figures 3.20 to 3.22 represent that signal recorded by electrometer from group of rods is quite weak as compared to the single rod, the reason is quite obvious that all the visible light generated by group of rods is not entering the fiber and majority of the signal is lost in the gel. Another reason may be the numerical aperture of optical fiber is smaller than the rods, hence it will not be able to collect the generated Cherenkov signal completely. The scatter fraction calculations for such a weak signal does not follow any trend and it requires further investigation to confirm any result. 


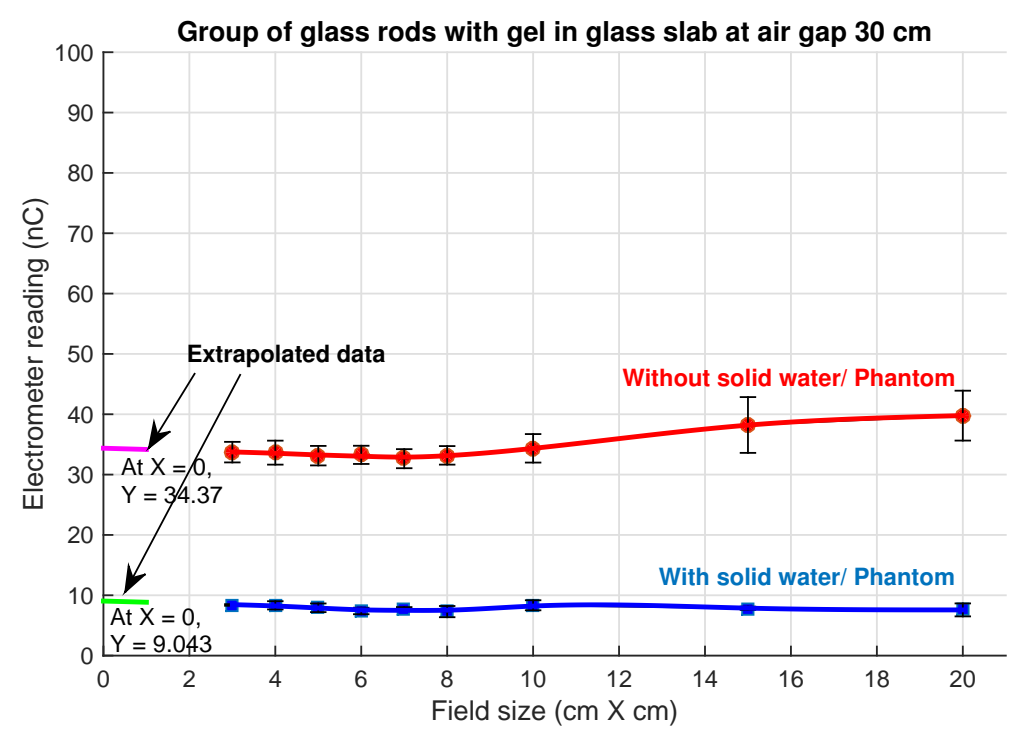

Figure 3.21: Cherenkov radiation from group of rods, at air gap $30 \mathrm{~cm}$

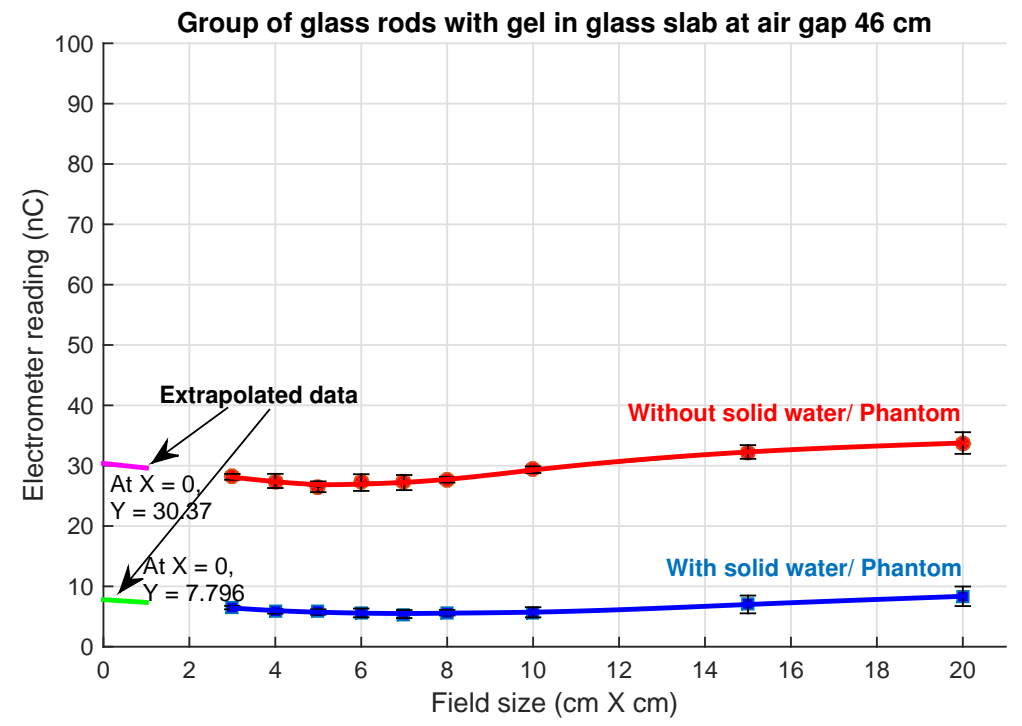

Figure 3.22: Cherenkov radiation from group of rods, at air gap $46 \mathrm{~cm}$ 


\section{Chapter 4}

\section{Conclusion}

\subsection{Conclusions}

In the present work, we built a prototype Cherenkov detector based on glass rods and investigated the response of prototype detector to scattered radiation in comparison with conventional detector based on ionization chamber. We found that for air gap of $10 \mathrm{~cm}$, the single glass rod based detector is less sensitive to scattered radiation than ion-chamber based detector.

For large air gaps, however, the results are not conclusive due to the fact that the signals from our Cherenkov prototype were very weak and uncertainties were quite large.

We also observed that polishing of glass rod ends and coupling of glass rod with the optical fiber plays a crucial role in transmitting and reproducing the signal.

Further investigation is required for multi-rod based detector.

\subsection{Future Work}

The anti-scatter detector that is proposed in this work is novel in its design and material, and could be very useful in clinical applications therefore there is a plenty of scope for future work that can be carried out in this direction.

Foremost, it is suggested to carry out theoretical simulations of this design so that validation of the experimental data can be done.

The improvement in the coupling can be the another direction in which work can be proceeded and instead of optical gel collimator can be used for transmitting the signal.

In this work, most of the study was carried out with the single glass rod, in future a prototype device with many glass rods can be developed and multi-pixel data can be recorded. 



\section{Appendix 1}

\section{MATLAB code for extrapolating the curves}

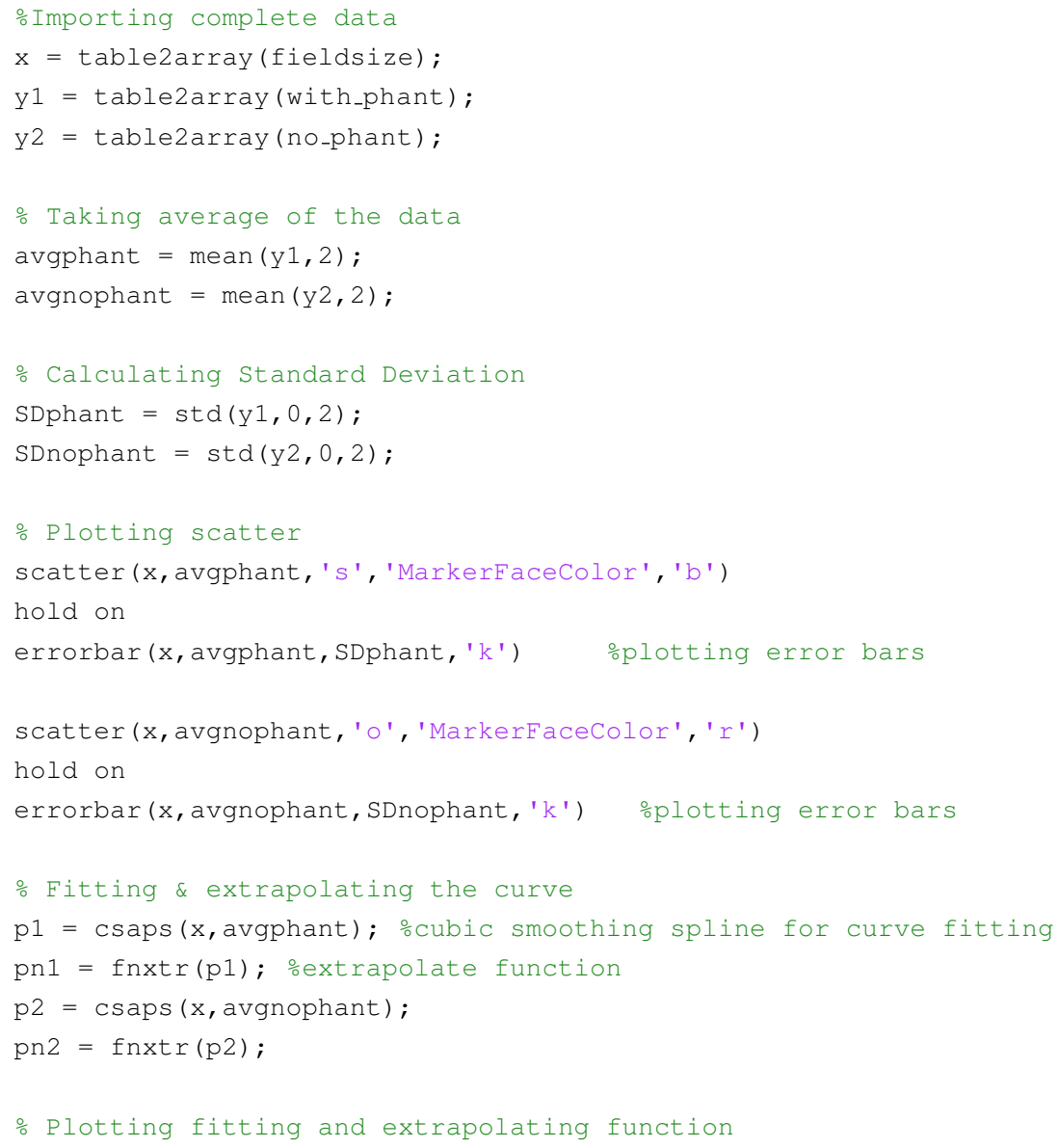




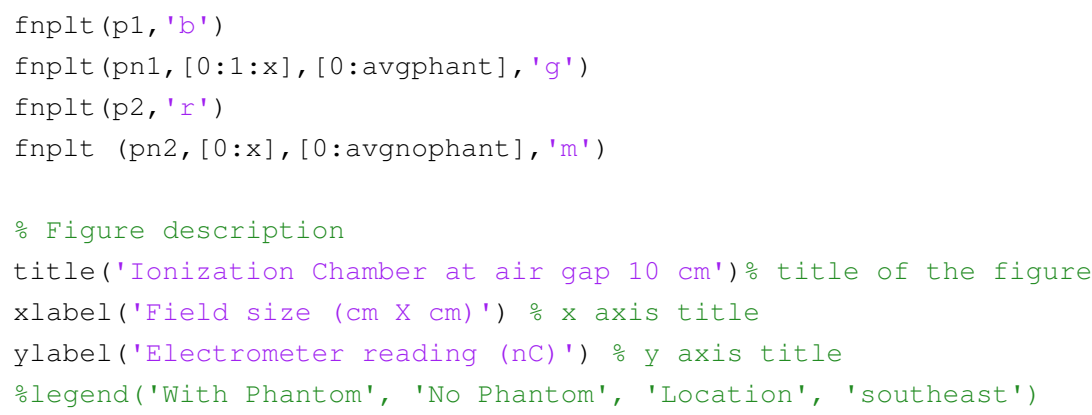




\section{Bibliography}

[1] P R Almond, P J Biggs, B M Coursey, W F Hanson, M Saiful Huq, R Nath, and D W O Rogers. Aapms tg-51 protocol for clinical reference dosimetry of high-energy photon and electron beams. Medical Physics, 1999.

[2] H. I. Amols, L. E. Reinstein, and B. Lagueux. A quantitative assessment of portal film contrast as a function of beam energy. Medical Physics, 13(5):711 - 716, September/ October 1986.

[3] A Assmus. Early history of x rays, 1995.

[4] J. P. Bissonnette, D. A. Jaffray, A. Fenster, and P. Munro. Optimal radiographic magnification for portal imaging. Medical Physics, 21(9):1435 - 1445, September 1994.

[5] A. L. Boyer, L. Antonuk, A. Fenster, M. Van Herk, H. Meertens, P. Munro, L. E. Reinstein, and J. Wong. A review of electronic portal imaging devices (epids). Medical Physics, 19(1):1 - 16, February 1992.

[6] P.A. Cerenkov. Visible radiation produced by electrons moving in a medium with velocities exceeding that of light. Physical Review, 52:378-381, 1937.

[7] M. A. Clift, R. A. Sutton, and D V Webb. Dealing with cerenkov radiation generated in organic scintillator dosimeters by bremsstrahlung beams. Physics in Medicine and Biology, 45:1165$1182,2000$.

[8] A. K. Glaser, J. M. Andreozzi, S. C. Davis, R. Zhang, B. W. Pogue, C. J. Fox, and D. J. Gladstone. Video-rate optical dosimetry and dynamic visualization of imrt and vmat treatment plans in water using cherenkov radiation. Medical Physics, 41(6):062102-1-062102-9, June 2014.

[9] A. K. Glaser, S. C. Davis, D. M. McClatchy, R. Zhang, B. W. Pogue, and D. J. Gladstone. Projection imaging of photon beams by the cerenkov effect. Medical Physics, 40(1):012101-1 - 012101-14, January 2013.

[10] A. G. Haus, R. E. Dickerson, K. E. Huff, S. Monte, B. A. Schlager, M. Atanas, and A. Matloubieh. Evaluation of a cassette-screen-film combination for radiation therapy portal localization imaging with improved contrast. Medical Physics, 24(10):1605 - 1608, October 1997.

[11] M. G. Herman, J. M. Balter, D. A. Jaffray, K. P. McGee, P. Munro, S. Shalev, M. V. Herk, and J. W. Wong. Clinical use of electronic portal imaging: Report of aapm radiation therapy committee task group 58. Medical Physics, 28(5):712 - 737, May 2001. 
[12] D. A. Jaffray, J. J. Battista, A. Fenster, and P. Munro. Xray scatter in megavoltage transmission radiography: Physical characteristics and influence on image quality. Medical Physics, 21(1):4560, January 1994.

[13] J. V. Jelly. Cerenkov radiation and its applications. British Journal of Applied Physics, 6:227 - 232, July 1955.

[14] J. V. Jelly. Cerenkov radiation and its applications. Pergamon Press, 1958.

[15] H E Johns and J R Cunningham. The Physics of Radiology. Charles C Thomas, Springfield, USA, fourth edition, 1983.

[16] F M Khan. The Physics of Radiation Therapy. Lippincott Williams \& Wilkins, Philadelphia, USA, third edition, 2003.

[17] K A Langmack. A review article portal imaging. The British Journal of Radiology, 74:789-804, 2001.

[18] D. W. Mah, D. M. Galbraith, and J. A. Rawlinson. Low-energy imaging with high-energy bremsstrahlung beams: Analysis and scatter reduction. Medical Physics, 20(3):653-665, MayJune 1993.

[19] B. M. C. McCurdy, K. Luchka, and S. Pistorius. Dosimetric investigation and portal dose image prediction using an amorphous silicon electronic portal imaging device. Medical Physics, 28(6):911 - 924, May 2001.

[20] X. Mei, J. A. Rowlands, and G. Pang. Electronic portal imaging based on cerenkov radiation: A new approach and its feasibility. Medical Physics, 33(11):4258 - 4270, November 2006.

[21] P Munro. Portal imaging technology: Past, present, and future. Seminars in Radiation Oncology,, 5(2):115-133, April 1995.

[22] G. Pang and J. A. Rowlands. Development of high quantum efficiency flat panel detectors for portal imaging: Intrinsic spatial resolution. Medical Physics, 29(10):2274 - 2285, October 2002.

[23] I. Silva and G. Pang. Characteristics of radiation induced light in optical fibres for portal imaging application. Radiation Physics and Chemistry, 81:599 - 608, 2012.

[24] A. Teymurazyan and G. Pang. Megavoltage x-ray imaging based on cerenkov effect: A new application of optical fibres to radiation therapy. International Journal of Optics, 2012:1-13, 2012.

[25] A. Teymurazyan and G. Pang. An inherent anti-scatter detector for megavoltage x-ray imaging. Physics in Medicine and Biology, 58:1479-1493, 2013.

[26] A. Teymurazyan, J. A. Rowlands, and G. Pang. Monte carlo simulation of a quantum noise limited cerenkov detector based on air-spaced light guiding taper for megavoltage x -ray imaging. Medical Physics, 41(4):1 -13, April 2014.

[27] S. A. Watson, M. Appleby, J. Klinger, S. Balzer, C. Lebeda, D. Bowman, J. Montoya, D. Bultman, C. Vecere, and C. Gossein. Design, fabrication and testing of a large anti-scatter grid for megavolt 
gamma-ray imaging. In IEEE Nuclear Science Symposium Conference Record, volume N14, page $148,2005$.

[28] A. Webb. Introduction to Biomedical Imaging. John Wiley \& Sons Inc., 2003.

[29] R. Zhang, A. K. Glaser, D. J. Gladstone, C. J. Fox, and B. W. Pogue. Superficial dosimetry imaging based on cerenkov emission for external beam radiotherapy with megavoltage x-ray beam. Medical Physics, 40(10):101914-1 - 101914-12, October 2013. 



\section{Index}

Air gap, 7

Cherenkov Radiation, 7

History, 7

Relation, 10

Theory, 8

Conclusions, 39

Correction Factors, 15

Data acquisition system, 18

Detector Quantum efficiency, 5

Experimental

Cherenkov setup, 17

Group of glass rods, 20

Methodology, 15

Setup, 16

Single glass rod, 17

Field size, 7

Future work, 39

Hypothesis, 13

Ionization Chamber, 15

Linear Accelerator (LINAC), 2

Literature review, 11
Machine head, 4

Objectives, 13

Phantom thickness, 7

Polishing, 18

Effect on Cherenkov signal, 30

Portal imaging, 5

Aspects, 5

CPID, 11

EPID, 11

Results, 21

Group of glass rods, 33

Ionization Chamber, 21, 24

Single glass rod, 30

Scatter fraction, 23

Scattering, 6

Factors, 7

Signal-to-Noise Ratio, 5

X-rays

Kilo-voltage, 1

Megavoltage, 2

Production, 1 